\title{
Differential equations and conformal structures
}

\author{
Paweł Nurowski \\ Instytut Fizyki Teoretycznej \\ Uniwersytet Warszawski \\ ul. Hoza 69, Warszawa \\ Poland \\ nurowski@fuw.edu.pl
}

September 6, 2018

\begin{abstract}
We provide five examples of conformal geometries which are naturally associated with ordinary differential equations (ODEs). The first example describes a one-to-one correspondence between the Wuenschmann class of 3rd order ODEs considered modulo contact transformations of variables and (local) 3-dimensional conformal Lorentzian geometries. The second example shows that every point equivalent class of 3rd order ODEs satisfying the Wuenschmann and the Cartan conditions define a 3-dimensional Lorentzian Einstein-Weyl geometry. The third example associates to each point equivalence class of 3rd order ODEs a 6-dimensional conformal geometry of neutral signature. The fourth example exhibits the one-to-one correspondence between point equivalent classes of 2nd order ODEs and 4-dimensional conformal Fefferman-like metrics of neutral signature. The fifth example shows the correspondence between undetermined ODEs of the Monge type and conformal geometries of signature $(3,2)$. The Cartan normal conformal connection for these geometries is reducible to the Cartan connection with values in the Lie algebra of the noncompact form of the exceptional group $G_{2}$. All the examples are deeply rooted in Elie Cartan's works on exterior differential systems.
\end{abstract}

MSC 2000: 34A26, 53B15, 53B30, 53B50.

Key words: differential geometry of ODEs, Cartan connections, noncompact form of the exceptional group G2. 


\section{Introduction}

One aspect of the Null Surface Formulation of General Relativity (NSF) of Fritelli, Kozameh and Newman [8] is to encode the conformal geometry of space-time in the geometry of a certain pair of partial differential equations (PDEs) on the plane. Although this pair of differential equations appears in NSF quite naturally, the question arises as to whether it is an accident or it is a feature of a deeper link between differential equations and conformal structures. A closer look at this question shows that the phenomenon observed in NSF is only a tip of an iceberg, and that there is an abundance of examples in which the geometry of differential equations can be related to the conformal geometry in various dimensions. The main aim of this paper is to describe these examples and to point out that all of them have their roots in Elie Cartan's works on differential systems.

The oldest and the simplest of these examples is due to Karl Wuenschmann. It is contained in his $\mathrm{PhD}$ dissertation [24] defended at the University of Greifswald in 1905. His result is quoted by Elie Cartan in a footnote of Ref. 3]. According to Cartan Wuenschmann observed that certain classes of 3rd order ordinary differential equations (ODEs) define, in a natural way, a conformal Lorentzian metric on the 3-dimensional spaces of their solutions. Chern in [5] interpreted the result of Wuenschmann in terms of a Cartan normal conformal connection [14 with values in the Lie algebra $\underline{\mathbf{s o}}(3,2)$. Recently, Newman and collaborators $[9$ proved that every 3-dimensional Lorentzian conformal geometry originates from a 3rd order ODE from the Wuenschmann class.

Although, due to Cartan, we have the precise coordinates of Wuenschmann thesis we were unable to get it from the University of Greifswald. Thus, we do not know how Wuenschmann obtained his result. In a joint paper 10 with Fritelli and Newman, we derived it by searching for 3rd ODEs for which it was possible to define a null separation between the solutions. We believe, that this derivation is very close to the Wuenschmann one. In the present paper, in Section 2, we give yet another derivation of Wuenschmann's result. This presentation is closely related to the description given in Cartan's footnote. In particular, we specify under which differential condition on $F=F\left(x, y, y^{\prime}, y^{\prime \prime}\right)$ the 3rd order ODE

$$
y^{\prime \prime \prime}=F\left(x, y, y^{\prime}, y^{\prime \prime}\right)
$$

is in the Wuenschmann class (condition (8)) and, using $F$ and its derivatives, we give the explicit formula for the conformal Lorentzian 3-metric. We also calculate the conformal invariants of these metrics, such as Cotton tensor, and relate them to the contact invariants of the corresponding ODEs from the Wuenschmann class. We end this section by providing nontrivial examples of ODEs from the Wuenschmann class.

Our next examples of conformal structures associated with differential equations are motivated by Cartan's paper [3]. In this paper Cartan studies the geometry of an ODE (11) given modulo the point transformation of variables. He shows that if, in addition to the Wuenschmann condition (8), the ODE satisfies another point invariant condition (17), then it defines a 3-dimensional Lorentzian Weyl geometry, i.e. the geometry defined by a conformal class of Lorentzian 3-metrics $[g]$ and a 1-form $[\nu]$ given up to a gradient. This Weyl geometry turns out to satisfy the Einstein-Weyl equations, which makes Cartan's observation important in the integrable systems theory (see e.g. 23]).

In Section 3 we formulate the equivalence problem for 3rd order ODEs considered modulo point transformations and present its solution (Theorem 3) due to Cartan [3]. We interpret the result in terms of Cartan's connection $\omega$ with values in the Lie algebra of a group $\mathbf{C O}(1,2) \rtimes \mathbf{R}^{3}$ - the semidirect product of the $\mathbf{S O}(1,2)$ group extended by the dilatations, and the translation group in $\mathbf{R}^{3}$. In case of a generic 3rd order ODE (II) this Cartan connection is defined on a principal $\mathbf{S O}(1,2)$ fiber 
bundle $\mathcal{P}$ over a certain four dimensional manifold but, if the equation satisfies the Wuenschmann condition (8) and the Cartan condition (17), it may be interpreted as a Cartan connection on a principal $\mathbf{C O}(1,2)$ fiber bundle over a three dimensional space identified with the solution space of (11). It is this special case which was studied by Cartan. In Section 3.1.1 we describe his result in the modern terminology. In particular, we explicitly write down the formualae for the metric $g_{e w}$ and the Weyl 1-form $\nu_{e w}$ in terms of function $F=F\left(x, y, y^{\prime}, y^{\prime \prime}\right)$ defining the equation. We also prove that the conditions (8) and (17) are equivalent to the Einstein-Weyl condition for the Weyl geometry $\left[g_{e w}, \nu_{e w}\right]$. The result is summarized in Theorem 4. In two examples (Example 2 and Example 3) we provide two nontrivial point equivalent classes of 3-rd order ODEs which satisfy conditions (8) and (17). The class of equations of Example 2 is a generalization of example (20) which was known to Tod [23]. Example 3 shows how to generate nontrivial $F=F\left(x, y, y^{\prime}, y^{\prime \prime}\right)$ satisfying (8) and (17) from particular solutions of reductions of the Einstein-Weyl geometries in 3-dimensions. Even very simple solutions, such as a solution $u=\sqrt{2 x}$ of the dKP equation (23), give rise to very nontrivial $F$ s (see formula (24)).

In Section 3.1.2 we return to the generic case of an ODE (1) given modulo point transformation and its Cartan connection $\omega$ on the $\mathbf{S O}(1,2)$ fiber bundle $\mathcal{P}$. We show that in this general case $\mathcal{P}$ is equipped with a special vector field whose integral curves foliate $\mathcal{P}$. The 6 -dimensional space of leaves of this foliation is naturally equipped with a conformal metric $[\tilde{\tilde{g}}]$ of signature $(3,3)$. This 6 -dimensional conformal structure encodes all the point invariant information about the point equivalent class of ODEs (11). In particular, the Cartan (point) invariants of Theorem 3 and the curvature of Cartan's connection $\omega$ can be equivalently described in terms of a Cartan normal conformal connection associated with the conformal class of metrics $[\tilde{g}]$. This result, which was not mentioned by Cartan, is summarized in Theorem 5; an explicit formula for this normal conformal connection is given by (25).

Section 4 deals with a geometry of a 2nd order ODE

$$
y^{\prime \prime}=Q\left(x, y, y^{\prime}\right)
$$

considered modulo point transformations of variables. It provides a next example of appearances of conformal geometry in the theory of differential equations. This case was studied by us in a joint paper with Sparling [17. In this paper, exploiting an analogy between 2nd order ODEs and 3 -dimensional CR-structures, we were able to associate a conformal 4-metric of signature $(2,2)$ with each point equivalence class of ODEs (2). The construction of this metric, described in [17, was motivated by Fefferman's construction [7] of Lorentzian metrics on a circle bundle over nondegenerate 3-dimensional CR-structures. Cartan, who formulated and solved the equivalence problem for ODEs (2) given modulo point transformations in his famous paper [2, overlooked existence of this metric. In Ref. 17, we showed that the conformal class of Fefferman-like $(2,2)$ signature metrics associated with a point equivalence class of ODEs (2) encodes all the point invariant information of such class. We summarize these results in Theorem 6 .

In Section 5 which, in our opinion, is the highlight of the paper, we consider the geometry of an undetermined equation

$$
z^{\prime}=F\left(x, y, y^{\prime}, y^{\prime \prime}, z\right)
$$

for two real functions $y=y(x)$ and $z=z(x)$ of one variable. The studies of equations of this type can be traced back to Gaspard Monge, who knew that every solution to the equation of the form

$$
z^{\prime}=F\left(x, y, y^{\prime}, z\right)
$$


was expressible without integrals by means of an arbitrary function of one variable and its derivatives. Hilbert [12, on an example of equation

$$
z^{\prime}=\left(y^{\prime \prime}\right)^{2},
$$

showed that, in general, equations (3) do not have this property. This result impressed Cartan, who previously (Ref. 4) considered equations (3) as equations for Cauchy characteristics of pairs of PDEs in the involution defined on the plane. Cartan solved the equivalence problem for these PDEs which, implicitly, solves an associated equivalence problem for ODEs (3). From Cartan's solution of this equivalence problem it follows that among equations (3) only those for which $F_{y^{\prime \prime}} y^{\prime \prime}=0$ have general solutions which can be expressed without integrals.

From the geometric point of view equations (3) for which $F_{y^{\prime \prime} y^{\prime \prime}} \neq 0$ are much more interesting then those with $F_{y^{\prime \prime} y^{\prime \prime}}=0$. It follows from Cartan's work [4] that nonequivalent classes of equations (3) with $F_{y^{\prime \prime} y^{\prime \prime}} \neq 0$ are distinguished by means of a curvature of a certain Cartan connection. Surprisingly, this connection has values in the Lie algebra of the noncompact form $\tilde{G}_{2}$ of the exceptional group $G_{2}$. The curvature of this connection is vanishing precisely in the case of equations equivalent to the Hilbert example (5). This, in particular means that the symmetry group of a very simple equation (5) is isomorphic to $\tilde{G}_{2}$. This fact, noticed with pride by Cartan in [4, was perhaps the first geometric realization of this group predicted to exist by Cartan and Engel in 1894.

The main original part of Section 5 consists in an observation that this Cartan connection can be understood as a reduction of a certain Cartan normal conformal connection. This is associated with a conformal metric $G_{(3,2)}$ of signature $(3,2)$ naturally defined by (3) on a 5 -dimensional space $J$ parametrized by the five independent variables $\left(x, y, y^{\prime}, y^{\prime \prime}, z\right)$. It follows that all the invariant information about the ODE (3) satisfying $F_{y^{\prime \prime} y^{\prime \prime}} \neq 0$ is encoded in the conformal properties of the metric $G_{(3,2)}$. We introduced this metric motivated by the Fefferman construction described in [17. Surprisingly, its existence, like the existence of Fefferman-like metrics described in Section 4, was overlooked by Cartan.

Section 5 has three subsections. The first one makes precise the notion of an equation having a general solution without integrals. It also contains the proof of Monge's result on equation (4) quoted above. The proof uses Cartan's method of equivalence [18] and aims to motivate the definition of equivalence problem for equations (3). This definition is given in Section 5.2 in terms of an equivalence of a system of three 1-forms (42) on $J$. The beginning of Section 5.3 reformulates Cartan's solution for the equivalence problem for pairs of PDEs in involution on the plane adapting it to the equivalence problem for ODEs (3) with $F_{y^{\prime \prime} y^{\prime \prime}} \neq 0$. This is summarized in Theorem 8 . The interpretation of this result in terms of Cartan's $\underline{\underline{g}}_{2}$-valued connection $\omega_{\tilde{G}_{2}}$ is given by formula (52). The rest of this section is devoted to the introduction and the discussion of a 5 -dimensional conformal $(3,2)$-signature metric whose Cartan normal conformal connection is reducible to $\omega_{\tilde{G}_{2}}$. This metric is defined by formula (53) and is finally expressible entirely in terms of the function $F=F\left(x, y, y^{\prime}, y^{\prime \prime}, z\right)$ and its derivatives in formula (54). The main properties of this metric are summarized in Theorem 9.

As an application of this section, in Example 6, we consider equations of the form

$$
z^{\prime}=F\left(y^{\prime \prime}\right)
$$

This generalizes (5). We show that in this case there is only one basic invariant of such equations. The metrics $G_{(3,2)}$ of Example 6 turn out to be always conformal to Einstein metrics. We characterize the Einstein scale for them by means of a simple ODE. Finally, in case of a generic $F$, we show that 
the square of the Weyl tensor for metrics $G_{(3,2)}$ can be interpreted in terms of a classical invariant of a certain polynomial of the fourth order. This polynomial resembles very much the Weyl tensor polynomial known in the Newman-Penrose formalism [16].

\section{Third order ODEs considered modulo contact transforma- tions}

In 1905 Wuenschmann [24] observed that the spaces of solutions of a certain class of 3rd order ODEs are naturally equipped with conformal Lorentzian geometries. His observation can be summarized as follows.

Consider a 3rd order ordinary differential equation

$$
y^{\prime \prime \prime}=F\left(x, y, y^{\prime}, y^{\prime \prime}\right)
$$

for a real function $y=y(x)$ of one variable. To simplify notation let $p=y^{\prime}$ and $q=y^{\prime \prime}$. Now, consider the four-dimensional space $J^{2}$ parametrized by $(x, y, p, q)$. This space, the second jet space, is a natural arena to study the geometry of equation (6). In particular, the total differential vector field

$$
\mathcal{D}=\partial_{x}+p \partial_{y}+q \partial_{p}+F \partial_{q}
$$

on $J^{2}$ yields the basic information about the solutions of (6). The integral curves of $\mathcal{D}$ foliate $J^{2}$ with 1-dimensional leaves. The leaf space $\mathcal{S}$ of this foliation is 3-dimensional and can be identified with the 3-dimensional space of solutions of (6). Following Chern [5] we equip $J^{2}$ with the following bilinear form ${ }^{1}$

$$
\tilde{g}=2[\mathrm{~d} y-p \mathrm{~d} x]\left[\mathrm{d} q-\frac{1}{3} F_{q} \mathrm{~d} p+K \mathrm{~d} y+\left(\frac{1}{3} q F_{q}-F-p K\right) \mathrm{d} x\right]-[\mathrm{d} p-q \mathrm{~d} x]^{2} .
$$

where

$$
K \equiv \frac{1}{6} \mathcal{D} F_{q}-\frac{1}{9} F_{q}^{2}-\frac{1}{2} F_{p}
$$

Clearly, this form is degenerate. It has signature $(+,-,-, 0)$ and its degenerate direction is tangent to the vector field $\mathcal{D}$. It is natural to ask about the transformation properties of $\tilde{g}$ under the Lie transport along the degenerate direction $\mathcal{D}$. It follows that $\tilde{g}$ transforms conformally under the Lie transport along $\mathcal{D}$ if and only if the function $F=F(x, y, p, q)$ defining the ODE satisfies the following nonlinear differential condition

$$
A \equiv F_{y}+\left(\mathcal{D}-\frac{2}{3} F_{q}\right) K=0 .
$$

This condition, the Wuenschmann condition defines the Wuenschmann class of 3rd order ODEs. Each equation from this class has a naturally defined conformal Lorentzian structure on the space of its solutions. In our description, if $F$ satisfies (8), this structure is obtained by projecting $\tilde{g}$ from $J^{2}$ to the leaf space $\mathcal{S}$ of integral lines of $\mathcal{D}$. Since in such case $\tilde{g}$ transforms conformally along $\mathcal{D}$, it projects to the conformal $(+,-,-)$ signature structure $[g]$ on $\mathcal{S}$. An interesting feature of the above Wuenschmann construction is its invariance under the contact transformations of the ODE (6). More precisely, if equation (6) undergoes a transformation of variables of the form

$$
x \rightarrow \bar{x}=\bar{x}(x, y, p), \quad y \rightarrow \bar{y}=\bar{y}(x, y, p), \quad p \rightarrow \bar{p}=\bar{p}(x, y, p),
$$

\footnotetext{
${ }^{1}$ Here and in the following we adapt the convention from General Relativity in which a symmetrized tensor product of two 1 -forms $\alpha$ and $\beta$ is denoted by $\alpha \beta=\frac{1}{2}(\alpha \otimes \beta+\beta \otimes \alpha)$, e.g. $\alpha^{2}=\alpha \otimes \alpha$.
} 
with

$$
\bar{y}_{x}-\bar{p} \bar{x}_{x}+p\left(\bar{y}_{y}-\bar{p} \bar{x}_{y}\right)=\bar{y}_{p}-\bar{p} \bar{x}_{p}=0
$$

then, if it is in the Wuenschmann class for the function $F=F(x, y, p, q)$, it is also in the Wuenschmann class for the transformed function $\bar{F}=\bar{F}(\bar{x}, \bar{y}, \bar{p}, \bar{q})$. It follows from the work of Chern [5] that the Wuenschmann condition is the lowest order contact invariant condition one can build out of $F$ and its partial derivatives. Moreover, every other contact invariant of an equation from the Wuenschmann class corresponds to a conformal invariant of the Lorentzian conformal structure $[g]$. These conformal invariants are constructed by means of the derivatives of the Cotton tensor $C$ of $[g]$. Assuming $A=0$ and using the explicit form of the projection $[g]$ of $\tilde{g}$ we calculate that the five independent components of $C$ are

$$
\begin{gathered}
C_{1}=F_{q q q q}, \quad C_{2}=K_{q q q}, \quad C_{3}=L_{q q}, \quad C_{4}=N_{q} \\
C_{5}=-3 K_{q q} L+3 K_{q} L_{q}-3 K L_{q q}+3 L_{q y}+3 N_{p}+F_{q} N_{q},
\end{gathered}
$$

where

$$
\begin{gathered}
L \equiv-\frac{1}{3} F_{q y}+\frac{1}{3} F_{q q} K-K_{p}-\frac{1}{3} F_{q} K_{q} \\
N \equiv \frac{1}{3} F_{q q} L-\frac{2}{3} F_{q} L_{q}-2 L_{p}+K K_{q q}-K_{q y}-\frac{1}{2} K_{q}^{2} .
\end{gathered}
$$

It is worth noting that the vanishing of $C_{1}$ implies the vanishing of all the $C_{i}$ s, so that the conformal structure $[g]$ has vanishing Cotton tensor iff $F_{q q q q}=0$. In such a case the corresponding Wuenschmann class of equations (6) is contact equivalent to the equation $y^{\prime \prime \prime}=0$.

Summing up we have the following theorem.

Theorem 1 (Wuenschmann)

Third order ODEs of the form

$$
y^{\prime \prime \prime}=F\left(x, y, y^{\prime}, y^{\prime \prime}\right)
$$

split onto two main contact nonequivalent classes, the Wuenschmann class and its complement. There are contact nonequivalent equations within the Wuenschmann class. Each representative of a contact equivalence class of equations satisfying Wuenschmann condition defines a conformal Lorentzian structure on the space of its solutions. The conformally equipped solution spaces of contact equivalent equations are conformally related, so that each equivalence class of equations for which $A=0$ has a natural 3-dimensional conformal Lorentzian structure associated with it.

There is a converse to this theorem.

Theorem 2 (Frittelli, Kozameh, Newman)

Every 3-dimensional Lorentzian conformal structure $[g]$ defines a contact equivalence class of third order ODEs satisfying the Wuenschmann condition.

Below, without the proof which can be found in [9], we sketch how to pass from $[g]$ to the associated class of ODEs. 
Given a conformal family of Lorentzian metrics $[g]$ on a 3-dimensional manifold $\mathcal{M}$ we start with a particular representative $g$ of $[g]$. This, in local coordinates $\left\{x^{i}\right\}, i=1,2,3$, can be written as $g=g_{i j} \mathrm{~d} x^{i} \mathrm{~d} x^{j}$. Since the metric $g$ is Lorentzian it is meaningful to consider the eikonal equation

$$
g^{i j} \frac{\partial S}{\partial x^{i}} \frac{\partial S}{\partial x^{j}}=0,
$$

for the real-valued function $S=S\left(x^{i}\right)$ on $\mathcal{M}$. This equation, being homogeneous in $S$, has the complete solution $S=S\left(x^{i} ; s\right)$ depending on a single parameter $s$. Now, treating $x^{i} \mathrm{~s}, i=1,2,3$ as constant parameters and $s$ as an independent variable we eliminate $x^{1}, x^{2}$ and $x^{3}$ by triple differentiation of equation $S=S\left(x^{i} ; s\right)$ with respect to $s$. As a result we get a relation of the form

$$
S^{\prime \prime \prime}=F\left(s, S, S^{\prime}, S^{\prime \prime}\right),
$$

which shows that $S=S(s)$ satisfies an ODE of the 3rd order. It follows that this equation satisfies the Wuenschmann condition (8). It also follows that if we start with another representative $\bar{g}$ of $[g]$ and find the corresponding complete solution $\bar{S}=\bar{S}\left(\bar{x}^{i} ; \bar{s}\right)$ of the corresponding eikonal equation we get a 3rd order ODE for $\bar{S}=\bar{S}(\bar{s})$ which is related to (9) by a contact transformation of variables $s, S$ and $S^{\prime}$.

\section{Example 1}

It can be easily checked that

$$
F(x, y, p, q)=\alpha \frac{\left[q^{2}+\left(1-p^{2}\right)^{2}\right]^{\frac{3}{2}}}{\left[1-p^{2}\right]^{\frac{3}{2}}}-3 \frac{p q^{2}}{1-p^{2}}-p\left(1-p^{2}\right)
$$

satisfies the Wuenschmann condition (8) for all values of the real parameter $\alpha$. Moreover, the 3rd order ODEs $y^{\prime \prime \prime}=F\left(x, y, y^{\prime}, y^{\prime \prime}\right)$ corresponding to different values of $\alpha>0$ are contact nonequivalent. It follows that the conformal Lorentzian structures $[g]$ associated with such $F$ s have 4 -dimensional group of conformal symmetries, which correspond to four contact symmetries of the associated 3rd order ODE [13].

\section{Third order ODEs considered modulo point transforma- tions}

Cartan [3] considered 3rd order ODEs modulo point transformations of variables. These transformations are more restrictive then the contact transformations. They merely mix the independent and dependent variables

$$
x \rightarrow \bar{x}=\bar{x}(x, y), \quad y \rightarrow \bar{y}=\bar{y}(x, y)
$$

of the equation (6). Cartan in [3] found a full set of invariants which determine whether two 3rd order ODEs are transformable to each other by a point transformation of variables. He used his equivalence method. This method starts with a system of four 1-forms

$$
\begin{gathered}
\omega^{1}=\mathrm{d} y-p \mathrm{~d} x \\
\omega^{2}=\mathrm{d} p-q \mathrm{~d} x \\
\omega^{3}=\mathrm{d} q-F(x, y, p, q) \mathrm{d} x \\
\omega^{4}=\mathrm{d} x
\end{gathered}
$$


which an ODE of the form (6) defines on the second jet space $J^{2}$. Under transformations (10) of the ODE (6) the forms (11) transform by

$$
\begin{gathered}
\omega^{1} \rightarrow \bar{\omega}^{1}=\alpha \omega^{1} \\
\omega^{2} \rightarrow \bar{\omega}^{2}=\beta\left(\omega^{2}+\gamma \omega^{1}\right) \\
\omega^{3} \rightarrow \bar{\omega}^{3}=\epsilon\left(\omega^{3}+\lambda \omega^{2}+\mu \omega^{1}\right) \\
\omega^{4} \rightarrow \bar{\omega}^{4}=\nu\left(\omega^{4}+\sigma \omega^{1}\right),
\end{gathered}
$$

where $\alpha, \beta, \gamma, \epsilon, \lambda, \mu, \nu, \sigma$ are functions on $J^{2}$ such that $\alpha \beta \epsilon \nu \neq 0$. These functions are determined by each particular choice of point transformation (10). Instead of working with forms $\left(\omega^{i}\right), i=1,2,3,4$, which are defined on $J^{2}$ only up to transformations (12), Cartan considers a manifold parametrized by $(x, y, p, q, \alpha, \beta, \gamma, \epsilon, \lambda, \mu, \nu, \sigma)$ and forms

$$
\begin{gathered}
\theta^{1}=\alpha \omega^{1} \\
\theta^{2}=\beta\left(\omega^{2}+\gamma \omega^{1}\right) \\
\theta^{3}=\epsilon\left(\omega^{3}+\lambda \omega^{2}+\mu \omega^{1}\right) \\
\theta^{4}=\nu\left(\omega^{4}+\sigma \omega^{1}\right),
\end{gathered}
$$

which are well defined there. Using his equivalence method he constructs a 7-dimensional manifold $\mathcal{P}$ on which the four forms $\theta^{1}, \theta^{2}, \theta^{3}, \theta^{4}$ supplemented by three other forms $\Omega_{1}, \Omega_{2}$ and $\Omega_{3}$ constitute a rigid coframe. This coframe encodes all the point invariant information about the ODE (6). More precisely, Cartan proves the following theorem.

\section{Theorem 3}

A $3 r d$ order ODE (6) considered modulo point transformations of variables (10) uniquely defines

- a 7-dimensional manifold $\mathcal{P}$,

- seven 1-forms $\theta^{1}, \theta^{2}, \theta^{3}, \theta^{4}, \Omega_{1}, \Omega_{2}, \Omega_{3}$ on $\mathcal{P}$ such that $\theta^{1} \wedge \theta^{2} \wedge \theta^{3} \wedge \theta^{4} \wedge \Omega_{1} \wedge \Omega_{2} \wedge \Omega_{3} \neq 0$ and

- functions $A, B, C, D, G, H, K, L, M, N$ on $\mathcal{P}$,

which satisfy the following differential conditions

$$
\begin{gathered}
\mathrm{d} \theta^{1}=\Omega_{1} \wedge \theta^{1}+\theta^{4} \wedge \theta^{2} \\
\mathrm{~d} \theta^{2}=\Omega_{2} \wedge \theta^{2}+\Omega_{3} \wedge \theta^{1}+\theta^{4} \wedge \theta^{3} \\
\mathrm{~d} \theta^{3}=\left(2 \Omega_{2}-\Omega_{1}\right) \wedge \theta^{3}+\Omega_{3} \wedge \theta^{2}+A \theta^{4} \theta^{1} \\
\mathrm{~d} \theta^{4}=\left(\Omega_{1}-\Omega_{2}\right) \wedge \theta^{4}+B \theta^{2} \wedge \theta^{1}+C \theta^{3} \wedge \theta^{1} \\
\mathrm{~d} \Omega_{1}=-\Omega_{3} \wedge \theta^{4}+(H+D) \theta^{1} \wedge \theta^{2}+(3 K-2 B) \theta^{1} \wedge \theta^{3}+(G+L) \theta^{1} \wedge \theta^{4}-C \theta^{2} \wedge \theta^{3} \\
\mathrm{~d} \Omega_{2}=D \theta^{1} \wedge \theta^{2}+2(K-B) \theta^{1} \wedge \theta^{3}+G \theta^{1} \wedge \theta^{4}-2 C \theta^{2} \wedge \theta^{3} \\
\mathrm{~d} \Omega_{3}=\left(\Omega_{2}-\Omega_{1}\right) \wedge \Omega_{3}+M \theta^{1} \wedge \theta^{2}+(D-H) \theta^{1} \wedge \theta^{3}+N \theta^{1} \wedge \theta^{4}+(2 K-B) \theta^{2} \wedge \theta^{3}+G \theta^{2} \wedge \theta^{4} .
\end{gathered}
$$


Two third order ODEs $y^{\prime \prime \prime}=F\left(x, y, y^{\prime}, y^{\prime \prime}\right)$ and $\bar{y}^{\prime \prime \prime}=\bar{F}\left(\bar{x}, \bar{y}, \bar{y}^{\prime}, \bar{y}^{\prime \prime}\right)$ are transformable to each other by means of a point transformation (10) if and only if there exists a diffeomorphism $\phi: \mathcal{P} \rightarrow \overline{\mathcal{P}}$ of the corresponding manifolds $\mathcal{P}$ and $\overline{\mathcal{P}}$ such that $\phi^{*}\left(\bar{\theta}^{i}\right)=\theta^{i}, i=1,2,3,4$, and $\phi^{*}\left(\bar{\Omega}_{\mu}\right)=\Omega_{\mu}$, $\mu=1,2,3$.

\subsection{Cartan connections associated with 3rd order ODEs considered mod- ulo point transformations}

Among the equivalence classes of 3rd order ODEs described by Theorem 3 there is a particularly simple class corresponding to the vanishing of all the functions $A, B, C, D, G, H, K, L, M, N$. In case of such ODEs the corresponding forms $\left(\theta^{1}, \theta^{2}, \theta^{3}, \theta^{4}, \Omega_{1}, \Omega_{2}, \Omega_{3}\right)$ can be considered a basis of left invariant forms on a Lie group which naturally identifies with the space $\mathcal{P}$. The structure constants of this group are determined by equations (13) with all the functions $A, B, C, D, G, H, K, L, M, N$ vanishing. This group turns out to be locally isomorphic to $\mathbf{C O}(1,2) \rtimes \mathbf{R}^{3}$, the semidirect product of the $\mathbf{S O}(1,2)$ group extended by the dilatations, and the translation group in $\mathbf{R}^{3}$. In this sense, Theorem 3 can be interpreted in terms of a $\mathbf{C O}(1,2) \rtimes \mathbf{R}^{3}$ Cartan connection defined over the space $J^{2}$. Explicitly, the 1-form

$$
\omega=\left(\begin{array}{ccccc}
\Omega_{2} & 0 & 0 & 0 & 0 \\
\theta^{1} & \Omega_{2}-\Omega_{1} & -\theta^{4} & 0 & 0 \\
\theta^{2} & -\Omega_{3} & 0 & -\theta^{4} & 0 \\
\theta^{3} & 0 & -\Omega_{3} & \Omega_{1}-\Omega_{2} & 0 \\
0 & \theta^{3} & -\theta^{2} & \theta^{1} & -\Omega_{2}
\end{array}\right),
$$

which has values in the Lie algebra of $\mathbf{C O}(1,2) \rtimes \mathbf{R}^{3}$, defines a Cartan connection on $\mathcal{P}$. To see this it is enough to observe that the system (13) guarantees that the annihilator of forms $\left(\theta^{1}, \theta^{2}, \theta^{3}, \theta^{4}\right)$ is integrable, so that $\mathcal{P}$ is fibered over the 4 -dimensional space of leaves tangent to this annihilator. This space of leaves naturally identifies with $J^{2}$. Using equations (13) and calculating

$$
R=\mathrm{d} \omega+\omega \wedge \omega
$$

to be

$$
R=\left(\begin{array}{ccccc}
\mathcal{F} & 0 & 0 & 0 & 0 \\
0 & R^{1}{ }_{1} & R^{1}{ }_{2} & 0 & 0 \\
0 & R^{2}{ }_{1} & 0 & R_{2}^{1} & 0 \\
\Theta^{3} & 0 & R^{2}{ }_{1} & -R^{1} & 0 \\
0 & \Theta^{3} & 0 & 0 & -\mathcal{F}
\end{array}\right)
$$

with

$$
\begin{gathered}
\mathcal{F}=D \theta^{1} \wedge \theta^{2}+2(K-B) \theta^{1} \wedge \theta^{3}-2 C \theta^{2} \wedge \theta^{3}+G \theta^{1} \wedge \theta^{4} \\
\Theta^{3}=-A \theta^{1} \wedge \theta^{4} \\
R_{1}^{1}=-H \theta^{1} \wedge \theta^{2}-K \theta^{1} \wedge \theta^{3}-C \theta^{2} \wedge \theta^{3}-L \theta^{1} \wedge \theta^{4} \\
R_{1}^{2}=-M \theta^{1} \wedge \theta^{2}+(H-D) \theta^{1} \wedge \theta^{3}+(B-2 K) \theta^{2} \wedge \theta^{3}-N \theta^{1} \wedge \theta^{4}-G \theta^{2} \wedge \theta^{4} \\
R_{2}{ }_{2}=B \theta^{1} \wedge \theta^{2}+C \theta^{1} \wedge \theta^{3},
\end{gathered}
$$


we find that $\mathbf{S O}(1,2) \rightarrow \mathcal{P} \rightarrow J^{2}$ equipped with $\omega$ is a Cartan bundle with a $\mathbf{C O}(1,2) \rtimes \mathbf{R}^{3}$ connection over $J^{2}$.

In the next subsection we discuss under which conditions Theorem 3 can be interpreted in terms of a Cartan connection over a certain 3-dimensional space, the space with which all the solution spaces of point equivalent equations (6) may be identified.

\subsubsection{A subclass defining Lorentzian Einstein-Weyl geometries on the solution space}

First, the system (13) guarantees that, not only $\mathcal{P}$ is foliated by the 3 -dimensional leaves discussed so far, but it is also foliated by 4-dimensional leaves. These are tangent to the integrable distribution on which the forms $\left(\theta^{1}, \theta^{2}, \theta^{3}\right)$ vanish. Thus $\pi: \mathcal{P} \rightarrow \mathcal{M}$ can be considered a fibre bundle over the 3dimensional space $\mathcal{M}$ of leaves of this foliation. A 4-dimensional group $\mathbf{C O}(1,2)$ acts naturally on the fibres $\pi^{-1}(\mathcal{M})$ of $\mathcal{P}$ equipping it with a structure of a $\mathbf{C O}(1,2)$ fibre bundle over $\mathcal{M}$. Now, the form $\omega$ defined by (14) can be interpreted as a $\mathbf{C O}(1,2) \rtimes \mathbf{R}^{3}$ Cartan connection on $\mathbf{C O}(1,2) \rightarrow \mathcal{P} \rightarrow \mathcal{M}$ iff in the curvature $R$ there are only horizontal $\theta^{1} \wedge \theta^{2}, \theta^{1} \wedge \theta^{3}$ and $\theta^{2} \wedge \theta^{3}$ terms. This is only possible if

$$
\text { a) } \quad A \equiv 0
$$

and

$$
\text { b) } \quad G \equiv 0 .
$$

These are also sufficient conditions since, if they are satisfied, the functions $N$ and $L$ also vanish. Vanishing of each of $A$ and $G$ is a point invariant property of the ODE (6). One can also consider these conditions independently of each other. The vanishing of $A$ is precisely the Wuenschmann condition (8) which, being contact invariant, is also a point invariant. If the equation (6) satisfies this condition it defines the conformal metric $g$ on $\mathcal{M}$. This conformal Lorentzian structure on $\mathcal{M}$ is the projection of the bilinear form

$$
\tilde{g}=2 \theta^{1} \theta^{3}-\left(\theta^{2}\right)^{2}
$$

from $\mathcal{P}$ to $\mathcal{M}$. Thus, similarly to the contact case, point equivalent classes of equations ([6) satisfying the Wuenschmann condition $A=0$ define a conformal structure on the space $\mathcal{M}$. If, in addition condition $b)$ is satisfied then the pair $\left(\tilde{g}, \tilde{\nu}=-2 \Omega_{2}\right)$ projects to a well defined Weyl geometry $\left[\left(g_{\text {ew }}, \nu_{\text {ew }}\right)\right]$ on the space $\mathcal{M}$. We recall that a Weyl geometry on a 3 -dimensional manifold $\mathcal{M}$ is the geometry associated with an equivalence class $[(g, \nu)]$ of pairs $(g, \nu)$, in which $g$ is a Lorentzian metric, $\nu$ is a 1-form, and two pairs $(g, \nu)$ and $\left(g^{\prime}, \nu^{\prime}\right)$ are in the equivalence relation iff there exists a function $\phi$ on $\mathcal{M}$ such that $g^{\prime}=\mathrm{e}^{-2 \phi} g$ and $\nu^{\prime}=\nu+2 \mathrm{~d} \phi$.

To see how the Weyl geometry $\left[\left(g_{e w}, \nu_{e w}\right)\right]$ appears in the above context we first remark that the condition $G \equiv 0$, when written in terms of the function $F=F(x, y, p, q)$ defining the equation ([6), is

$$
G \equiv 0 \quad \Longleftrightarrow \quad \mathcal{D}^{2} F_{q q}-\mathcal{D} F_{q p}+F_{q y}=0 .
$$

Then, identifying $\mathcal{M}$ with the quotient $J^{2} / \mathcal{D}$ and using the $(x, y, p, q)$ coordinates on $J^{2}$, we have

$$
\begin{aligned}
& \tilde{g}=\beta^{2}\left[2 \omega^{1}\left(\omega^{3}-\frac{1}{3} F_{q} \omega^{2}+\left(\frac{1}{6} \mathcal{D} F_{q}-\frac{1}{2} F_{p}-\frac{1}{9} F_{q}^{2}\right) \omega^{1}\right)-\left(\omega^{2}\right)^{2}\right] \\
& -\tilde{\nu}=2 \Omega_{2}=2 \mathrm{~d} \log \beta+\frac{2}{3}\left(F_{q p}-\mathcal{D} F_{q q}\right) \omega^{1}+\frac{2}{3} F_{q q} \omega^{2}+\frac{2}{3} F_{q} \omega^{4} .
\end{aligned}
$$


The bilinear form $\tilde{g}$ is identical with (7), thus due to the Wuenschmann condition $A=0$, it projects to a conformal structure $\left[g_{e w}\right]$ on $\mathcal{M}$. Calculating the Lie derivative of $\tilde{\nu}$ with respect to $\mathcal{D}$ we find that

$$
\mathcal{L}_{\mathcal{D}} \tilde{\nu}=\frac{2}{3}\left(\mathcal{D}^{2} F_{q q}-\mathcal{D} F_{q p}+F_{q y}\right) \omega^{1}+\mathrm{d}(\ldots) .
$$

Thus, due to condition (17), $\mathcal{L}_{\mathcal{D}} \tilde{\nu}$ is a total differential. This means that $\tilde{\nu}$ projects to a class of 1-forms $\left[\nu_{e w}\right]$ on $\mathcal{M}$ which are given up to an addition of a gradient.

It follows that the so defined Weyl geometry $\left[\left(g_{e w}, \nu_{e w}\right)\right]$ on $\mathcal{M}$ satisfies the Einstein-Weyl equations. To see this we first recall that a 3-dimensional Weyl geometry $\left[\left(g=g_{i j} \theta^{i} \theta^{j}, \nu\right)\right]$ defines a Weyl connection, which is totally determined by the connection 1 -forms $\Gamma_{j}^{i}$ satisfying

$$
\mathrm{d} \theta^{i}+\Gamma_{j}^{i} \wedge \theta^{j}=0, \quad \mathrm{~d} g_{i j}-\Gamma_{i j}-\Gamma_{j i}=-\nu g_{i j}, \quad \Gamma_{i j}=g_{i k} \Gamma_{j}^{k} .
$$

The Weyl geometry is said to be Einstein-Weyl iff the curvature

$$
\Omega^{i}{ }_{j}=\frac{1}{2} R^{i}{ }_{j k l} \theta^{k} \wedge \theta^{l}=\mathrm{d} \Gamma^{i}{ }_{j}+\Gamma_{k}^{i} \wedge \Gamma_{j}^{k}
$$

of this connection satisfies

$$
R_{(i j)}-\frac{1}{3} R g_{i j}=0
$$

where

$$
R_{j k}=R_{j i k}^{i} \quad R_{(i j)}=\frac{1}{2}\left(R_{i j}+R_{j i}\right)
$$

and $R=g^{i j} R_{i j}, g^{i k} g_{k j}=\delta^{i}{ }_{j}$.

It follows that in the case of Weyl geometry $\left[\left(g_{e w}, \nu_{e w}\right)\right]$ the Einstein-Weyl condition (18) reduces to the requirement that the point invariant $M$ of the system (13) vanishes. To show that conditions $A=G=0$, which were needed to define $\left[\left(g_{e w}, \nu_{e w}\right)\right]$, imply $M=0$ we apply the exterior derivative $\mathrm{d}$ to the both sides of equations (13). Then from the equation $\mathrm{d}^{2} \theta^{3}=0$ we deduce that $N=L=0$. Having this and insisting on $\mathrm{d}^{2} \Omega_{2}=0$ we get that $D=2 H$, which is only possible if $M=0$.

Summarizing we have the following theorem.

Theorem 4 (Cartan)

A point equivalence class of 3rd order ODEs represented by an ODE

$$
y^{\prime \prime \prime}=F\left(x, y, y^{\prime}, y^{\prime \prime}\right)
$$

which satisfies Wuenschmann condition (8) and Cartan condition (17) defines a Lorentzian EinsteinWeyl geometry $\left[\left(g_{e w}, \nu_{e w}\right)\right]$ on the 3-dimensional space $\mathcal{M}$. This space can be identified with the solution space of any of the ODEs from the equivalence class.

It is a nontrivial task to find $F=F(x, y, p, q)$ which satisfies the Einstein-Weyl conditions (87), (17). Cartan gave several examples of such $F$ s (see [23] for a discussion of that issue). Here we present two other ways of constructing them.

\section{Example 2}

It is relatively easy to find all point equivalence classes of 3rd order ODEs which admit at least four infinitesimal point symmetries [13]. Among them there is a 1-parameter family of nonequivalent ODEs represented by

$$
F=\frac{\left(\sqrt{a\left(2 q y-p^{2}\right)}\right)^{3}}{y^{2}}
$$


which corresponds to nonequivalent Einstein-Weyl geometries for each value of the real constant $a$. This constant enumerates nonequivalent ODEs; its sign is correlated with the sign of $\left(2 q y-p^{2}\right)$, so that the expression under the square root is positive. If $a \rightarrow \infty$ the equivalence class of ODEs may be represented by

$$
F=q^{3 / 2},
$$

which also satisfies conditions (8), (17).

\section{Example 3}

Since 3-dimensional Lorentzian Einstein-Weyl geometries are known to be generated by solutions of various integrable systems, one can try to use such solutions to associate with them point equivalence classes of ODEs (6). We illustrate this procedure on an example of solutions to the dKP equation.

The dKP equation for a real function $u=u(x, y, t)$ can be considered to be the Froebenius condition

$$
\begin{aligned}
& \mathrm{d} \bar{\omega}^{1} \wedge \bar{\omega}^{1} \wedge \bar{\omega}^{4}=0 \\
& \mathrm{~d} \bar{\omega}^{4} \wedge \bar{\omega}^{1} \wedge \bar{\omega}^{4}=0
\end{aligned}
$$

for the two Pfaffian forms

$$
\begin{gathered}
\bar{\omega}^{1}=\mathrm{d} x+\left(u+v^{2}\right) \mathrm{d} t+v \mathrm{~d} y \\
\bar{\omega}^{4}=\mathrm{d} v-\left(u_{y}+u_{x} v\right) \mathrm{d} t-u_{x} \mathrm{~d} y
\end{gathered}
$$

in a 4-dimensional space parametrized by $(x, y, t, v)$. Indeed, by substitution of (22) to (21) we find that (21) is equivalent to

$$
u_{y y}=-\left(u_{x}\right)^{2}+u_{x t}-u u_{x x},
$$

which is the dKP equation. Since every solution to (23) generates a 3-dimensional Lorentzian Einstein-Weyl geometry [6] it is reasonable to ask if there is a point equivalence class of 3rd order ODEs associated with each such solution. It turns out that the answer to this question is positive. Given a solution $u=u(x, y, t)$ of the dKP equation there is a point equivalence class of 3rd order ODEs, with a representative in the form (6), such that the four 1 -forms $\left(\bar{\omega}^{1}, \bar{\omega}^{2}, \bar{\omega}^{3}, \bar{\omega}^{4}\right)$ of (12) encoding it have, in a convenient coordinate system $(x, y, t, v)$ on $J^{2}$, representatives $\bar{\omega}^{1}$ and $\bar{\omega}^{4}$ of (22) and $\bar{\omega}^{2}$ and $\bar{\omega}^{3}$ given by

$$
\begin{gathered}
\bar{\omega}^{2}=\left(-u u_{x x}-2 u_{x y} v+u_{x x} v^{2}\right) \mathrm{d} t-u_{x x} \mathrm{~d} x-u_{x y} \mathrm{~d} y \\
\bar{\omega}^{3}=\left(-u u_{x x}^{2}-4 u_{x y}^{2}+4 u_{x x} u_{x y} v-u_{x x}^{2} v^{2}\right) \mathrm{d} t-u_{x x}^{2} \mathrm{~d} x+u_{x x}\left(-2 u_{x y}+u_{x x} v\right) \mathrm{d} y .
\end{gathered}
$$

In particular, equations (21) guarantee that there exists a coordinate $X$ on $J^{2}$ such that in the class (12) of forms $\bar{\omega}^{4}$ there is an exact form $\mathrm{d} X$. This defines a function $X$, which in turn is interpreted as the independent variable of the associated ODE. For example, for a very simple solution

$$
u=\sqrt{2 x}
$$

of the dKP equation we find that

$$
X=t+\frac{1}{2} v^{2}+\sqrt{2 x},
$$

which enables us to find the associated class of 3rd order ODEs. This class may be represented by quite a nontrivial

$$
F(x, y, p, q)=\frac{p q(-12+3 p q-8 \sqrt{1-p q})+8(1+\sqrt{1-p q})}{p^{3}} .
$$


It can be checked by a direct substitution that such $F$ satisfies the Einstein-Weyl conditions (8), (17).

We close this section with a remark, that it is not clear whether all 3-dimensional Lorentzian EinsteinWeyl geometries have their associated point equivalence classes of 3rd order ODEs. Our experience, based on the Cartan's equivalence method, suggests that it is very likely.

\subsubsection{Conformal metric of signature $(3,3)$ associated with a point equivalence class of 3rd order ODEs}

If an ODE (6) does not satisfy the Wuenschmann condition (15), it is impossible to define a conformal structure in 3 dimensions out of the Cartan invariants (13). However, irrespectively of the Wuenschmann condition (15) being satisfied or not, with each point equivalence class of ODEs [6], we can associate a conformal metric of signature $(+,+,+,-,-,-)$, whose conformal invariants encode all the point invariant information about the corresponding class of ODEs. We achieve this by using Sparling's procedure 22] which, with 'the Levi-Civita part'

$$
\Gamma=\left(\Gamma_{j}^{i}\right)=\left(\begin{array}{ccc}
\Omega_{2}-\Omega_{1} & -\theta^{4} & 0 \\
-\Omega_{3} & 0 & -\theta^{4} \\
0 & -\Omega_{3} & \Omega_{1}-\Omega_{2}
\end{array}\right),
$$

of the Cartan connection (14) and with the bilinear form $\tilde{g}=g_{i j} \theta^{i} \theta^{j}$ of (16), associates a new bilinear form

$$
\tilde{\tilde{g}}=\epsilon_{i j k} \theta^{i} \Gamma^{j}{ }_{l} g^{l k}
$$

on $\mathcal{P}$. Here

$$
\left(g^{i j}\right)=\left(\begin{array}{ccc}
0 & 0 & 1 \\
0 & -1 & 0 \\
1 & 0 & 0
\end{array}\right)
$$

and $\epsilon_{i j k}$ is the standard Levi-Civita symbol in $\mathbf{R}^{3}$ so that

$$
\tilde{\tilde{g}}=2\left[\left(\Omega_{1}-\Omega_{2}\right) \theta^{2}-\Omega_{3} \theta^{1}+\theta^{4} \theta^{3}\right] .
$$

This bilinear form is degenerate on $\mathcal{P}$ and has $(+,+,+,-,-,-, 0)$ signature. Denoting the basis of vector fields on $\mathcal{P}$ dual to the 1 -forms $\left(\theta^{1}, \theta^{2}, \theta^{3}, \theta^{4}, \Omega_{1}, \Omega_{2}, \Omega_{3}\right)$ by $\left(X_{1}, X_{2}, X_{3}, X_{4}, Y_{1}, Y_{2}, Y_{3}\right)$, we find that the degenerate direction of $\tilde{\tilde{g}}$ is tangent to the vector field $Z=Y_{1}+Y_{2}$.

It is remarkable that, due to equations (13), the bilinear form $\tilde{\tilde{g}}$ transforms conformally when Lie transported along $Z$. Explicitly, without any assumptions on the Cartan invariants $A, B, C, D$, $G, H, K, L, M, N$, we have

$$
\mathcal{L}_{Z} \tilde{\tilde{g}}=\tilde{\tilde{g}}
$$

Thus, the bilinear form $\tilde{\tilde{g}}$ naturally descends to a conformal metric $g_{\mathcal{N}}$ of neutral signature on the 6-dimensional space $\mathcal{N}$ of integral curves of the vector field $Z$. This conformal metric yields all the point invariant information about the corresponding point equivalent class of ODEs (6). In particular, the Cartan invariants $A, B, C, D, G, H, K, L, M, N$ can be understood as curvature coefficients of the Cartan normal conformal connection associated with $g_{\mathcal{N}}$. This Cartan connection 
can be represented by the following so(4,4)-valued 1 -form

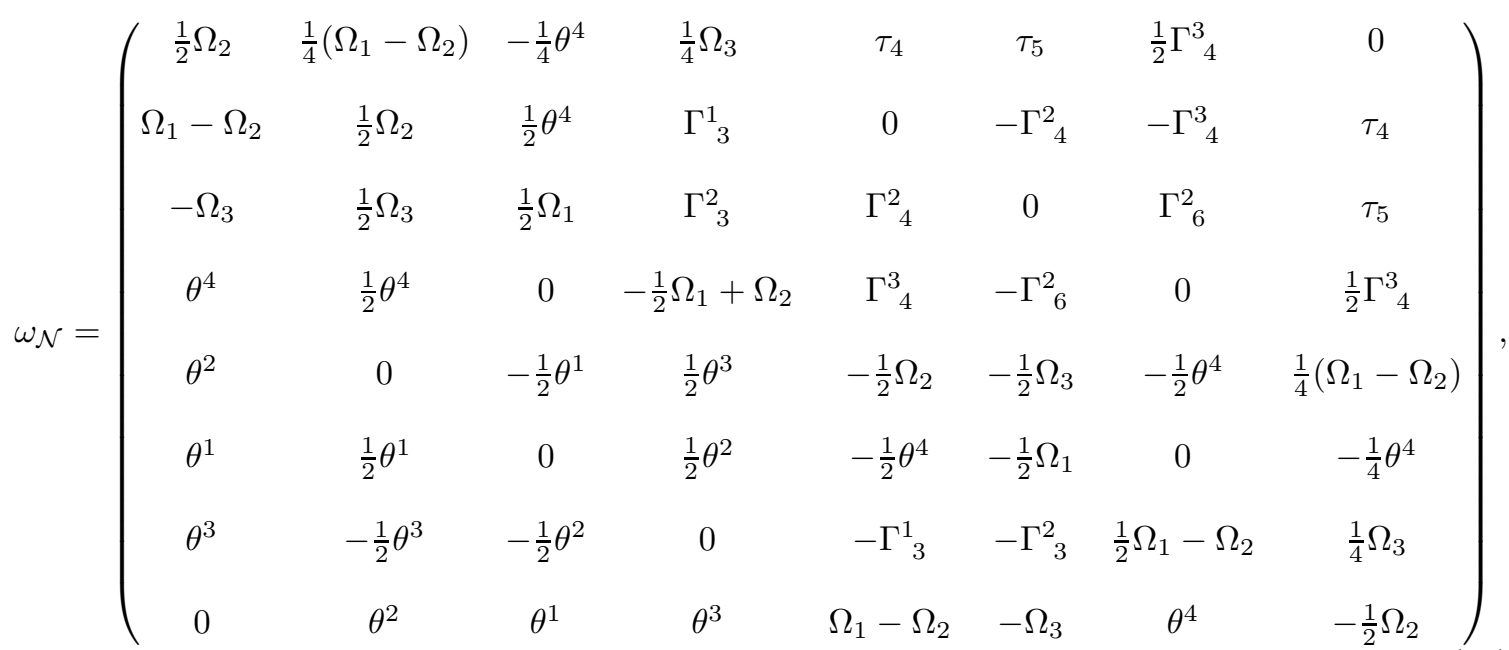

where

$$
\begin{gathered}
\tau_{4}=\frac{1}{12}\left[X_{3}(G)-6 H\right] \theta^{1}-\frac{1}{4} K \theta^{2}-\frac{1}{2} C \theta^{3} \\
\tau_{5}=\frac{1}{2}\left[-A C-2 X_{2}(L)-2 M+X_{4}(D)\right] \theta^{1}+\frac{1}{12}\left[X_{3}(G)-6 H+6 D\right] \theta^{2}+\frac{1}{4}(-2 B+3 K) \theta^{3}+\frac{1}{2} G \theta^{4} \\
\Gamma_{3}^{1}=\frac{1}{2} \Omega_{3}+\frac{1}{2}(G-L) \theta^{1} \\
\Gamma_{3}^{2}=N \theta^{1}+\frac{1}{2}(G-L) \theta^{2}+A \theta^{4} \\
\Gamma_{4}^{2}=M \theta^{1}-H \theta^{2}+\frac{1}{2}(2 B-3 K) \theta^{3}-\frac{1}{2}(G+L) \theta^{4} \\
\Gamma_{6}^{2}=(-H+D) \theta^{1}+\frac{1}{2} K \theta^{2}+C \theta^{3} \\
\Gamma_{4}^{3}=\frac{1}{2}(2 B-K) \theta^{1}+C \theta^{2}
\end{gathered}
$$

on $\mathcal{P}$.

We remark that not all 6 -dimensional split-signature conformal metrics originate from a point equivalence 3rd order ODEs. To see this, we calculate the curvature

$$
R_{\mathcal{N}}=\mathrm{d} \omega_{\mathcal{N}}+\omega_{\mathcal{N}} \wedge \omega_{\mathcal{N}}
$$

of $\omega_{\mathcal{N}}$ and observe ${ }^{2}$ that it has quite special form when compared to the curvature of Cartan's normal conformal connection associated with a generic $(+,+,+,-,-,-)$ signature metric.

Summarizing, we have the following theorem.

\section{Theorem 5}

Each point equivalence class of $3 r d$ order ODEs

$$
y^{\prime \prime \prime}=F\left(x, y, y^{\prime}, y^{\prime \prime}\right)
$$

defines a conformal split-signature metric $g_{\mathcal{N}}$ on a 6 -dimensional manifold $\mathcal{N}$, which is canonically associated with this class of ODEs. The conformal metric $g_{\mathcal{N}}$ yields all the point invariant information about the corresponding class of 3 rd order ODEs.

\footnotetext{
${ }^{2}$ We omit writing down the explicit formulae for this curvature here.
} 


\section{Second order ODEs considered modulo point transforma- tions}

This case has been recently carefully studied in Ref. 17]. The ODE part of this paper includes, in particular, description of the geometry associated with an equation

$$
y^{\prime \prime}=Q\left(x, y, y^{\prime}\right)
$$

considered modulo point transformations (10). This geometry, in the convenient parametrization $\left(x, y, p=y^{\prime}\right)$ of the first jet space $J^{1}$, turns out to be very closely related to the geometry associated with the following split signature metric, the Fefferman metric,

$$
g=2\left[(\mathrm{~d} p-Q \mathrm{~d} x) \mathrm{d} x-(\mathrm{d} y-p \mathrm{~d} x)\left(\mathrm{d} \phi+\frac{2}{3} Q_{p} \mathrm{~d} x+\frac{1}{6} Q_{p p}(\mathrm{~d} y-p \mathrm{~d} x)\right)\right]
$$

on $J^{1} \times \mathbf{R}$. More precisely, we have the following theorem.

\section{Theorem 6}

1) Every second order ODE (26) endows its corresponding space $J^{1} \times \mathbf{R}$ with an orientation and with the Fefferman metric 27).

2) If the ODE undergoes a point transformation (10) then its Fefferman metric transforms conformally.

3) All the point invariants of a point equivalence class of ODEs (26) are expressible in terms of the conformal invariants of the associated conformal class of Fefferman metrics.

4) The Fefferman metrics (27) are very special among all the split signature metrics on 4-manifolds. Their Weyl tensor has algebraic type $(N, N)$ in the Cartan-Petrov-Penrose classification [1, 19, 20, 21. Both, the selfdual $C^{+}$and the antiselfdual $C^{-}$, parts of it are expressible in terms of only one component. In fact, $C^{+}$is proportional to

$$
w_{1}=D^{2} Q_{p p}-4 D Q_{p y}-D Q_{p p} Q_{p}+4 Q_{p} Q_{p y}-3 Q_{p p} Q_{y}+6 Q_{y y}
$$

and $C^{-}$is proportional to

$$
w_{2}=Q_{p p p p}
$$

where

$$
D=\partial_{x}+p \partial_{y}+Q \partial_{p} .
$$

Each of the conditions $w_{1}=0$ and $w_{2}=0$ is invariant under point transformations (10).

5) Cartan normal conformal connection associated with any conformal class [g] of Fefferman metrics is reducible to a certain $\mathbf{S L}(2+1, \mathbf{R})$ connection naturally defined on an 8-dimensional bundle over $J^{1}$ which, via Cartan's equivalence method, is uniquely associated with the point equivalence class of corresponding ODEs (26). The curvature of this connection has very simple form

$$
\Omega \sim\left(\begin{array}{ccc}
0 & w_{2} & * \\
0 & 0 & w_{1} \\
0 & 0 & 0
\end{array}\right) .
$$


If $w_{1}=0$ or $w_{2}=0$ this connection can be further understood as a Cartan normal projective connection over a certain two dimensional space $S$ equipped with a projective structure [15]. $S$ can be identified either with the solution space of the ODE (26) in the $w_{1}=0$ case, or with the solution space of its dual ${ }^{3} O D E$ in the $w_{2}=0$ case.

\section{Equations $z^{\prime}=F\left(x, y, y^{\prime}, y^{\prime \prime}, z\right)$, noncompact form of the ex- ceptional group $G_{2}$ and conformal metrics of signature $(3,2)$}

\subsection{Equations with integral-free solutions}

Consider a differential equation of the form

$$
G\left(x, y, y^{\prime}, \ldots, y^{(m)}, z, z^{\prime}, \ldots, z^{(k)}\right)=0
$$

for real functions $y=y(x)$ and $z=z(x)$ of one real variable. In this equation $G: \mathbf{R}^{m+k+3} \rightarrow \mathbf{R}$ and $y^{(r)}, z^{(q)}$ denote the $r$ th and the $q$ th derivative of $y$ and $z$ with respect to $x$. In 1912 Hilbert 12 considered a subclass of equations (28) which he called equations with integral-free solutions (Germ. integrallose Aufloesungen). These equations are defined as follows.

Definition 1 Equation (28) has integral-free solutions iff its general solution can be written as

$$
\begin{aligned}
& x=x\left(t, w(t), w^{\prime}(t), \ldots, w^{(p)}(t)\right) \\
& y=y\left(t, w(t), w^{\prime}(t), \ldots, w^{(p)}(t)\right) \\
& z=z\left(t, w(t), w^{\prime}(t), \ldots, w^{(p)}(t)\right),
\end{aligned}
$$

where $w=w(t)$ is an arbitrary sufficiently smooth real function of one real variable.

As an example consider equation

$$
z^{\prime}=y .
$$

Clearly $x=t, z=w(t), y=w^{\prime}(t)$ is its general solution, which shows that (29) is in the Hilbert class of equations with integral-free solutions. Very simple equation (29) belongs to the class of first order Monge equations

$$
z^{\prime}=F\left(x, y, y^{\prime}, z\right),
$$

which are equations (28) with unknowns of at most of the first order.

Associated with each first order Monge equation (30) there is a 4-dimensional space $J$ parametrized by $(x, y, p, z)$ and two 1 -forms

$$
\begin{gathered}
\omega^{1}=\mathrm{d} z-F(x, y, p, z) \mathrm{d} x \\
\omega^{2}=\mathrm{d} y-p \mathrm{~d} x .
\end{gathered}
$$

Every solution of the Monge equation (30) is a curve $c(t)=(x(t), y(t), p(t), z(t))$ in $J$ on which the forms $\omega^{1}$ and $\omega^{2}$ vanish.

Suppose now, that given a Monge equation (30), there exists a transformation of the associated variables $(x, y, p, z)$

$$
\left(\begin{array}{l}
x \\
y \\
p \\
z
\end{array}\right) \stackrel{\phi}{\rightarrow}\left(\begin{array}{l}
\bar{x} \\
\bar{y} \\
\bar{p} \\
\bar{z}
\end{array}\right)=\left(\begin{array}{l}
\bar{x}(x, y, p, z) \\
\bar{y}(x, y, p, z) \\
\bar{p}(x, y, p, z) \\
\bar{z}(x, y, p, z)
\end{array}\right)
$$

\footnotetext{
${ }^{3}$ See e.g. [17 for the concept of dual second order ODEs.
} 
such that

$$
\begin{aligned}
\mathrm{d} \bar{y}-\bar{p} \mathrm{~d} \bar{x} & =\alpha \omega^{1}+\beta \omega^{2} \\
\mathrm{~d} \bar{p}-\bar{z} \mathrm{~d} \bar{x} & =\gamma \omega^{1}+\delta \omega^{2},
\end{aligned}
$$

with $\alpha, \beta, \gamma, \delta$ functions on $J$ satisfying $\Delta=\alpha \delta-\beta \gamma \neq 0$. In such case

$$
\begin{gathered}
\omega^{1}=\Delta^{-1}[\delta(\mathrm{d} \bar{y}-\bar{p} \mathrm{~d} \bar{x})-\beta(\mathrm{d} \bar{p}-\bar{z} \mathrm{~d} \bar{x})] \\
\omega^{2}=\Delta^{-1}[-\gamma(\mathrm{d} \bar{y}-\bar{p} \mathrm{~d} \bar{x})+\alpha(\mathrm{d} \bar{p}-\bar{z} \mathrm{~d} \bar{x})] .
\end{gathered}
$$

Thus, taking

$$
\bar{x}=t, \quad \bar{y}=w(t), \quad \bar{p}=w^{\prime}(t), \quad \bar{z}=w^{\prime \prime}(t)
$$

we construct a curve in $J$ on which the forms $\omega^{1}$ and $\omega^{2}$ identically vanish. Now, the inverse of $\phi$ which gives $x=x(\bar{x}, \bar{y}, \bar{p}, \bar{z})$, etc., provides

$$
\begin{aligned}
& x=x\left(t, w(t), w^{\prime}(t), w^{\prime \prime}(t)\right) \\
& y=y\left(t, w(t), w^{\prime}(t), w^{\prime \prime}(t)\right) \\
& z=z\left(t, w(t), w^{\prime}(t), w^{\prime \prime}(t)\right),
\end{aligned}
$$

which is an integral-free solution of the Monge equation (30).

We summarize our discussion in the following Lemma.

\section{Lemma 1}

Every first order Monge equation (30) admitting coordinate transformation (31) which realizes (32) has integral-free solutions.

\section{Example 4}

Consider equation

$$
z^{\prime}=\left(y^{\prime}\right)^{2} .
$$

Its corresponding forms are $\omega^{1}=\mathrm{d} z-p^{2} \mathrm{~d} x, \omega^{2}=\mathrm{d} y-p \mathrm{~d} x$. The change of variables $x=\frac{1}{2} \bar{z}$, $y=\frac{1}{2}(\bar{z} \bar{x}-\bar{p}), \quad z=\frac{1}{2} \bar{z} \bar{x}^{2}-\bar{p} \bar{x}+\bar{y}, \quad p=\bar{x}$ brings them to the form $\omega^{1}=\mathrm{d} \bar{y}-\bar{p} \mathrm{~d} \bar{x}-\bar{x}(\mathrm{~d} \bar{p}-\bar{z} \mathrm{~d} \bar{x})$, $\omega^{2}=-\frac{1}{2}(\mathrm{~d} \bar{p}-\bar{z} \mathrm{~d} \bar{x})$. This proves that substitution (33) leads to the following integral-free solution of equation (34):

$$
\begin{gathered}
x=\frac{1}{2} w^{\prime \prime}(t) \\
y=\frac{1}{2} t w^{\prime \prime}(t)-\frac{1}{2} w^{\prime}(t) \\
z=\frac{1}{2} t^{2} w^{\prime \prime}(t)-t w^{\prime}(t)+w(t) .
\end{gathered}
$$

A natural question as to whether all the first order Monge equations have integral-free solutions was answered in affirmative by Monge. Thus, we have the following theorem.

Theorem 7 (Monge)

Every first order Monge equation has integral-free solutions.

It is instructive to sketch the proof of this theorem.

Given a Monge equation (30) we consider its associated two 1-forms

$$
\omega^{1}=\mathrm{d} z-F(x, y, p, z) \mathrm{d} x \quad \text { and } \quad \omega^{2}=\mathrm{d} y-p \mathrm{~d} x
$$


on $J$. We say that another pair of linearly independent 1 -forms $\left(\bar{\omega}^{1}, \bar{\omega}^{2}\right)$ on $J$ is equivalent to the pair (35) if there exists a transformation of variables (31) and functions $\alpha, \beta, \gamma, \delta, \alpha \delta-\beta \gamma \neq 0$, on $J$ such that

$$
\begin{aligned}
& \phi^{*}\left(\bar{\omega}^{1}\right)=\alpha \omega^{1}+\beta \omega^{2} \\
& \phi^{*}\left(\bar{\omega}^{2}\right)=\gamma \omega^{1}+\delta \omega^{2} .
\end{aligned}
$$

According to Lemma 1, if we were able to show that there is only one equivalence class of forms $\left(\omega^{1}, \omega^{2}\right)$ equivalent to $(\mathrm{d} \bar{y}-\bar{p} \mathrm{~d} \bar{x}, \mathrm{~d} \bar{p}-\bar{z} \mathrm{~d} \bar{x})$, the theorem would be proven. Thus, in the process of proving the Monge theorem, we are led to study the equivalence problem for two 1-forms given modulo transformations (36) on an open set of $\mathbf{R}^{4}$. Introducing the total differential vector field $D=\partial_{x}+p \partial_{y}+F \partial_{z}$ it is not difficult to prove that a pair of 1 -forms (35) originating from the Monge equations for which

$$
F_{p p}=0 \quad \text { and } \quad D F_{p}-F_{y}-F_{p} F_{z}=0
$$

and a pair of forms originating from the equations for which at least one of the above conditions is not satisfied are not equivalent. Then, the Cartan equivalence method applied to the forms related to the first order Monge equations not satisfying (37) shows that they are all locally equivalent to $(\mathrm{d} \bar{y}-\bar{p} \mathrm{~d} \bar{x}, \mathrm{~d} \bar{p}-\bar{z} \mathrm{~d} \bar{x})$. Thus, the first order Monge equations for which at least one of conditions (37) is not satisfied have general solutions of the form

$$
\begin{aligned}
& x=x\left(t, w(t), w^{\prime}(t), w^{\prime \prime}(t)\right) \\
& y=y\left(t, w(t), w^{\prime}(t), w^{\prime \prime}(t)\right) \\
& z=z\left(t, w(t), w^{\prime}(t), w^{\prime \prime}(t)\right) .
\end{aligned}
$$

On the other hand, if we apply the Cartan equivalence method to the forms originating from the Monge equations satisfying (37), we show that they are all locally equivalent to ( $\mathrm{d} \bar{z}, \mathrm{~d} \bar{y}-\bar{p} \mathrm{~d} \bar{x})$. Thus, taking $\bar{z}=$ const, $\bar{x}=t, \bar{y}=w(t)$ and $\bar{p}=w^{\prime}(t)$ we show that in such case the Monge equations have general solutions of the form

$$
\begin{aligned}
& x=x\left(t, w(t), w^{\prime}(t)\right) \\
& y=y\left(t, w(t), w^{\prime}(t)\right) \\
& z=z\left(t, w(t), w^{\prime}(t)\right) .
\end{aligned}
$$

Therefore in the both nonequivalent cases (38) and (39) the Monge equations have integral-free solutions. This finishes the proof of the Monge theorem.

Hilbert in 12 considered an equation

$$
z^{\prime}=\left(y^{\prime \prime}\right)^{2}
$$

and proved that it has not the property of having integral-free solutions. It turns out, that among all the equations which have not this property, the Hilbert equation (40) is, in a certain sense, the simplest one.

\subsection{Equivalence of forms associated with ODEs $z^{\prime}=F\left(x, y, y^{\prime}, y^{\prime \prime}, z\right)$}

The Hilbert equation (40) is a special case of an equation

$$
z^{\prime}=F\left(x, y, y^{\prime}, y^{\prime \prime}, z\right)
$$

Equations of this type were considered by Cartan 4 who, in particular, observed that they describe Cauchy characteristics of pairs of involutive second order PDEs for a real function of two variables. 
In the context of the present paper we are interested under what conditions equations (41) have integral-free solutions. The treatment of the problem is a simple generalization of the method described in the sketch of the proof of Monge's theorem. Thus, with each equation (41) we associate three 1-forms

$$
\begin{gathered}
\omega^{1}=\mathrm{d} z-F(x, y, p, q, z) \mathrm{d} x \\
\omega^{2}=\mathrm{d} y-p \mathrm{~d} x \\
\omega^{3}=\mathrm{d} p-q \mathrm{~d} x,
\end{gathered}
$$

which live on a 5 -dimensional manifold $J$ parametrized by $\left(x, y, p=y^{\prime}, q=y^{\prime \prime}, z\right)$. Following the case of Monge equations, we need to study the equivalence problem for the triples of linearly independent 1-forms $\left(\omega^{1}, \omega^{2}, \omega^{3}\right)$ on an open set of $\mathbf{R}^{5}$. More precisely, let $\left(\omega^{1}, \omega^{2}, \omega^{3}\right)$ be defined on a open set $J \subset \mathbf{R}^{5}$ parametrized by $(x, y, p, q, z)$ and $\left(\bar{\omega}^{1}, \bar{\omega}^{2}, \bar{\omega}^{3}\right)$ be defined on a set $\bar{J} \subset \mathbf{R}^{5}$ parametrized by $(\bar{x}, \bar{y}, \bar{p}, \bar{q}, \bar{z})$. We say that the two triples $\left(\omega^{1}, \omega^{2}, \omega^{3}\right)$ and $\left(\bar{\omega}^{1}, \bar{\omega}^{2}, \bar{\omega}^{3}\right)$ are (locally) equivalent iff there exists a (local) diffeomorphism $\phi: J \rightarrow \bar{J}$

$$
\left(\begin{array}{l}
x \\
y \\
p \\
q \\
z
\end{array}\right) \stackrel{\phi}{\rightarrow}\left(\begin{array}{c}
\bar{x} \\
\bar{y} \\
\bar{p} \\
\bar{q} \\
\bar{z}
\end{array}\right)=\left(\begin{array}{l}
\bar{x}(x, y, p, q, z) \\
\bar{y}(x, y, p, q, z) \\
\bar{p}(x, y, p, q, z) \\
\bar{q}(x, y, p, q, z) \\
\bar{z}(x, y, p, q, z)
\end{array}\right)
$$

and a $\mathbf{G L}(3, \mathbf{R})$-valued function

$$
f=\left(\begin{array}{lll}
\alpha & \beta & \gamma \\
\delta & \epsilon & \lambda \\
\kappa & \mu & \nu
\end{array}\right)
$$

on $J$ such that

$$
\begin{gathered}
\phi^{*}\left(\bar{\omega}^{1}\right)=\alpha \omega^{1}+\beta \omega^{2}+\gamma \omega^{3} \\
\phi^{*}\left(\bar{\omega}^{2}\right)=\delta \omega^{1}+\epsilon \omega^{2}+\lambda \omega^{3} \\
\phi^{*}\left(\bar{\omega}^{3}\right)=\kappa \omega^{1}+\mu \omega^{2}+\nu \omega^{3} .
\end{gathered}
$$

The equivalence problem for such triples was solved by Cartan. His solution, in particular, can be applied to the triples of 1-forms (42) originating from the Cartan equations (41). Cartan's analysis, restricted to such triples, shows that they split onto two main nonequivalent classes. The first class originates from equations (41) satisfying

$$
F_{q q}=0
$$

the second class is defined by the equations for which

$$
F_{q q} \neq 0 .
$$

Both the above classes include nonequivalent triples of 1-forms, but only the first class originates from equations (41) with integral-free solution. All the Cartan equations with $F_{q q} \neq 0$ have not the property of having integral-free solutions. The Hilbert equation (40) is one of the equations from this class.

\section{Example 5}

According to the above discussion, if $k \neq 0$ and $k \neq 1$ equation

$$
z^{\prime}=\frac{1}{k}\left(y^{\prime \prime}\right)^{k}
$$


has not the property of having integral-free solutions. Thus, since one is forced to use integrals to write down the general solution of [45], we solve it by putting

$$
x=t, \quad y=w(t), \quad z=\frac{1}{k} \int w^{\prime \prime}(t)^{k} \mathrm{~d} t .
$$

Cartan found better solution

$$
\begin{gathered}
x=(k-1) t^{\frac{k-2}{k-1}} w^{\prime \prime}(t) \\
y=\frac{1}{2}(k-1)^{2} t^{\frac{2 k-3}{k-1}} w^{\prime \prime}(t)^{2}-(k-1) t^{\frac{k-2}{k-1}} w^{\prime}(t) w^{\prime \prime}(t)+\frac{1}{2}(k-1) \int t^{\frac{k-2}{k-1}} w^{\prime \prime}(t)^{2} \mathrm{~d} t \\
z=\frac{k-1}{k} t^{2} w^{\prime \prime}(t)-t w^{\prime}(t)+w(t) .
\end{gathered}
$$

We prefer this solution rather then (46) since it involves only second power of $w^{\prime \prime}$ under the integral, whereas the solution (46) involves the $k$ th power. This example shows that, for a given Cartan equation, among many different expressions for its general solution which involve integrals there could be some preferred ones. The precise meaning of this observation is worth further investigation.

\section{3 $\tilde{G}_{2}$ Cartan connection for equation $z^{\prime}=F\left(x, y, y^{\prime}, y^{\prime \prime}, z\right)$ and conformal $(3,2)$-signature geometry}

We will not comment any further on Cartan equations for which $F_{q q}=0$. Instead, we concentrate on much more interesting $F_{q q} \neq 0$ case.

First, we briefly sketch Cartan's results on equivalence problem for forms

$$
\begin{gathered}
\omega^{1}=\mathrm{d} z-F(x, y, p, q, z) \mathrm{d} x \\
\omega^{2}=\mathrm{d} y-p \mathrm{~d} x \\
\omega^{3}=\mathrm{d} p-q \mathrm{~d} x
\end{gathered}
$$

satisfying $F_{q q} \neq 0$. On doing that we supplement these forms to a coframe $\left(\omega^{1}, \omega^{2}, \omega^{3}, \omega^{4}, \omega^{5}\right)$ on the $(x, y, p, q, z)$ space such that

$$
\begin{gathered}
\omega^{1}=\mathrm{d} z-F(x, y, p, q, z) \mathrm{d} x \\
\omega^{2}=\mathrm{d} y-p \mathrm{~d} x \\
\omega^{3}=\mathrm{d} p-q \mathrm{~d} x \\
\omega^{4}=\mathrm{d} q \\
\omega^{5}=\mathrm{d} x .
\end{gathered}
$$

Since we are interested in all forms $\left(\omega^{1}, \omega^{2}, \omega^{3}\right)$ which are equivalent to the forms (47) via transformations (43)-(44) this coframe is not unique. It is given up to the following freedom:

$$
\left(\begin{array}{c}
\omega^{1} \\
\omega^{2} \\
\omega^{3} \\
\omega^{4} \\
\omega^{5}
\end{array}\right) \rightarrow\left(\begin{array}{c}
\bar{\omega}^{1} \\
\bar{\omega}^{2} \\
\bar{\omega}^{3} \\
\bar{\omega}^{4} \\
\bar{\omega}^{5}
\end{array}\right)=\left(\begin{array}{ccccc}
\alpha & \beta & \gamma & 0 & 0 \\
\delta & \epsilon & \lambda & 0 & 0 \\
\kappa & \mu & \nu & 0 & 0 \\
\pi & \rho & \sigma & \tau & \chi \\
\pi^{\prime} & \rho^{\prime} & \sigma^{\prime} & \tau^{\prime} & \chi^{\prime}
\end{array}\right)\left(\begin{array}{c}
\omega^{1} \\
\omega^{2} \\
\omega^{3} \\
\omega^{4} \\
\omega^{5}
\end{array}\right),
$$


which suggests that instead of working with a not uniquely defined coframe (48) on the $(x, y, p, q, z)$ space it is better to use five well defined linearly independent 1-forms

$$
\left(\begin{array}{c}
\theta^{1} \\
\theta^{2} \\
\theta^{3} \\
\theta^{4} \\
\theta^{5}
\end{array}\right)=\left(\begin{array}{ccccc}
\alpha & \beta & \gamma & 0 & 0 \\
\delta & \epsilon & \lambda & 0 & 0 \\
\kappa & \mu & \nu & 0 & 0 \\
\pi & \rho & \sigma & \tau & \chi \\
\pi^{\prime} & \rho^{\prime} & \sigma^{\prime} & \tau^{\prime} & \chi^{\prime}
\end{array}\right)\left(\begin{array}{c}
\omega^{1} \\
\omega^{2} \\
\omega^{3} \\
\omega^{4} \\
\omega^{5}
\end{array}\right)
$$

on a bigger space parametrized by $\left(x, y, p, q, z, \alpha, \beta, \gamma, \delta, \epsilon, \lambda, \kappa, \mu, \nu, \pi, \rho, \sigma, \tau, \chi, \pi^{\prime}, \rho^{\prime}, \sigma^{\prime}, \tau^{\prime}, \chi^{\prime}\right)$. Now, assuming that $F_{q q} \neq 0$ and using his equivalence method (which involved several reductions and prolongations ${ }^{4}$ ) Cartan was able to prove that on a certain 14-dimensional manifold $P$ the forms $\left(\theta^{1}, \theta^{2}, \theta^{3}, \theta^{4}, \theta^{5}\right)$ can be supplemented in a unique way to a unique coframe. More precisely, he proved the following theorem.

Theorem 8 (Cartan)

An equivalence class of forms

$$
\begin{gathered}
\omega^{1}=\mathrm{d} z-F(x, y, p, q, z) \mathrm{d} x \\
\omega^{2}=\mathrm{d} y-p \mathrm{~d} x \\
\omega^{3}=\mathrm{d} p-q \mathrm{~d} x,
\end{gathered}
$$

for which $F_{q q} \neq 0$, uniquely defines a 14-dimensional manifold $P$ and a preferred coframe $\left(\theta^{1}, \theta^{2}, \theta^{3}\right.$, $\left.\theta^{4}, \theta^{5}, \Omega_{1}, \Omega_{2}, \Omega_{3}, \Omega_{4}, \Omega_{5}, \Omega_{6}, \Omega_{7}, \Omega_{8}, \Omega_{9}\right)$ on it such that

$$
\begin{gathered}
\mathrm{d} \theta^{1}=\theta^{1} \wedge\left(2 \Omega_{1}+\Omega_{4}\right)+\theta^{2} \wedge \Omega_{2}+\theta^{3} \wedge \theta^{4} \\
\mathrm{~d} \theta^{2}=\theta^{1} \wedge \Omega_{3}+\theta^{2} \wedge\left(\Omega_{1}+2 \Omega_{4}\right)+\theta^{3} \wedge \theta^{5} \\
\mathrm{~d} \theta^{3}=\theta^{1} \wedge \Omega_{5}+\theta^{2} \wedge \Omega_{6}+\theta^{3} \wedge\left(\Omega_{1}+\Omega_{4}\right)+\theta^{4} \wedge \theta^{5} \\
\mathrm{~d} \theta^{4}=\theta^{1} \wedge \Omega_{7}+\frac{4}{3} \theta^{3} \wedge \Omega_{6}+\theta^{4} \wedge \Omega_{1}+\theta^{5} \wedge \Omega_{2} \\
\mathrm{~d} \theta^{5}=\theta^{2} \wedge \Omega_{7}-\frac{4}{3} \theta^{3} \wedge \Omega_{5}+\theta^{4} \wedge \Omega_{3}+\theta^{5} \wedge \Omega_{4} .
\end{gathered}
$$

Note that the above theorem implies formulae for the differentials of the forms $\Omega_{\mu}, \mu=1,2, \ldots, 9$.

\footnotetext{
${ }^{4}$ See e.g. in Ref. 18 for the definitions of these procedures
} 
Explicitly, these differentials are:

$$
\begin{aligned}
& \mathrm{d} \Omega_{1}=\Omega_{3} \wedge \Omega_{2}+\frac{1}{3} \theta^{3} \wedge \Omega_{7}-\frac{2}{3} \theta^{4} \wedge \Omega_{5}+\frac{1}{3} \theta^{5} \wedge \Omega_{6}+\theta^{1} \wedge \Omega_{8}+ \\
& \frac{3}{8} c_{2} \theta^{1} \wedge \theta^{2}+b_{2} \theta^{1} \wedge \theta^{3}+b_{3} \theta^{2} \wedge \theta^{3}+a_{2} \theta^{1} \wedge \theta^{4}+a_{3} \theta^{1} \wedge \theta^{5}+a_{3} \theta^{2} \wedge \theta^{4}+a_{4} \theta^{2} \wedge \theta^{5} \\
& \mathrm{~d} \Omega_{2}=\Omega_{2} \wedge\left(\Omega_{1}-\Omega_{4}\right)-\theta^{4} \wedge \Omega_{6}+\theta^{1} \wedge \Omega_{9}+ \\
& \frac{3}{8} c_{3} \theta^{1} \wedge \theta^{2}+b_{3} \theta^{1} \wedge \theta^{3}+a_{3} \theta^{1} \wedge \theta^{4}+a_{4} \theta^{1} \wedge \theta^{5}+b_{4} \theta^{2} \wedge \theta^{3}+a_{4} \theta^{2} \wedge \theta^{4}+a_{5} \theta^{2} \wedge \theta^{5} \\
& \mathrm{~d} \Omega_{3}=\Omega_{3} \wedge\left(\Omega_{4}-\Omega_{1}\right)-\theta^{5} \wedge \Omega_{5}+\theta^{2} \wedge \Omega_{8}- \\
& \frac{3}{8} c_{1} \theta^{1} \wedge \theta^{2}-b_{1} \theta^{1} \wedge \theta^{3}-a_{1} \theta^{1} \wedge \theta^{4}-a_{2} \theta^{1} \wedge \theta^{5}-b_{2} \theta^{2} \wedge \theta^{3}-a_{2} \theta^{2} \wedge \theta^{4}-a_{3} \theta^{2} \wedge \theta^{5} \\
& \mathrm{~d} \Omega_{4}=\Omega_{2} \wedge \Omega_{3}+\frac{1}{3} \theta^{3} \wedge \Omega_{7}+\frac{1}{3} \theta^{4} \wedge \Omega_{5}-\frac{2}{3} \theta^{5} \wedge \Omega_{6}+\theta^{2} \wedge \Omega_{9}- \\
& \frac{3}{8} c_{2} \theta^{1} \wedge \theta^{2}-b_{2} \theta^{1} \wedge \theta^{3}-a_{2} \theta^{1} \wedge \theta^{4}-a_{3} \theta^{1} \wedge \theta^{5}-b_{3} \theta^{2} \wedge \theta^{3}-a_{3} \theta^{2} \wedge \theta^{4}-a_{4} \theta^{2} \wedge \theta^{5} \\
& \mathrm{~d} \Omega_{5}=\Omega_{1} \wedge \Omega_{5}+\Omega_{3} \wedge \Omega_{6}-\theta^{5} \wedge \Omega_{7}+\theta^{3} \wedge \Omega_{8}+ \\
& \frac{9}{32} \delta_{1} \theta^{1} \wedge \theta^{2}+\frac{3}{4} c_{1} \theta^{1} \wedge \theta^{3}+\frac{3}{4} b_{1} \theta^{1} \wedge \theta^{4}+\frac{3}{4} b_{2} \theta^{1} \wedge \theta^{5}+\frac{3}{4} c_{2} \theta^{2} \wedge \theta^{3}+\frac{3}{4} b_{2} \theta^{2} \wedge \theta^{4}+\frac{3}{4} b_{3} \theta^{2} \wedge \theta^{5} \\
& \mathrm{~d} \Omega_{6}=\Omega_{2} \wedge \Omega_{5}+\Omega_{4} \wedge \Omega_{6}+\theta^{4} \wedge \Omega_{7}+\theta^{3} \wedge \Omega_{9}+ \\
& \frac{9}{32} \delta_{2} \theta^{1} \wedge \theta^{2}+\frac{3}{4} c_{2} \theta^{1} \wedge \theta^{3}+\frac{3}{4} b_{2} \theta^{1} \wedge \theta^{4}+\frac{3}{4} b_{3} \theta^{1} \wedge \theta^{5}+\frac{3}{4} c_{3} \theta^{2} \wedge \theta^{3}+\frac{3}{4} b_{3} \theta^{2} \wedge \theta^{4}+\frac{3}{4} b_{4} \theta^{2} \wedge \theta^{5} \\
& \mathrm{~d} \Omega_{7}=\frac{4}{3} \Omega_{5} \wedge \Omega_{6}+\left(\Omega_{1}+\Omega_{4}\right) \wedge \Omega_{7}+\theta^{4} \wedge \Omega_{8}+\theta^{5} \wedge \Omega_{9}+ \\
& \frac{9}{64} e \theta^{1} \wedge \theta^{2}-\frac{3}{8} \delta_{1} \theta^{1} \wedge \theta^{3}-\frac{3}{8} c_{1} \theta^{1} \wedge \theta^{4}-\frac{3}{8} c_{2} \theta^{1} \wedge \theta^{5}-\frac{3}{8} \delta_{2} \theta^{2} \wedge \theta^{3}-\frac{3}{8} c_{2} \theta^{2} \wedge \theta^{4}-\frac{3}{8} c_{3} \theta^{2} \wedge \theta^{5} \\
& \mathrm{~d} \Omega_{8}=\Omega_{5} \wedge \Omega_{7}+\left(2 \Omega_{1}+\Omega_{4}\right) \wedge \Omega_{8}+\Omega_{3} \wedge \Omega_{9}+ \\
& h_{1} \theta^{1} \wedge \theta^{2}+h_{2} \theta^{1} \wedge \theta^{3}+h_{3} \theta^{1} \wedge \theta^{4}+h_{4} \theta^{1} \wedge \theta^{5}+h_{5} \theta^{2} \wedge \theta^{3}+h_{4} \theta^{2} \wedge \theta^{4}+h_{6} \theta^{2} \wedge \theta^{5} \\
& \mathrm{~d} \Omega_{9}=\Omega_{6} \wedge \Omega_{7}+\left(\Omega_{1}+2 \Omega_{4}\right) \wedge \Omega_{9}+\Omega_{2} \wedge \Omega_{8}+ \\
& k_{1} \theta^{1} \wedge \theta^{2}+\frac{1}{32}\left(3 e+32 h_{5}\right) \theta^{1} \wedge \theta^{3}+\frac{1}{32}\left(-3 \delta_{1}+32 h_{4}\right) \theta^{1} \wedge \theta^{4}+ \\
& \frac{1}{32}\left(-3 \delta_{2}+32 h_{6}\right) \theta^{1} \wedge \theta^{5}+k_{2} \theta^{2} \wedge \theta^{3}+\frac{1}{32}\left(-3 \delta_{2}+32 h_{6}\right) \theta^{2} \wedge \theta^{4}+k_{3} \theta^{2} \wedge \theta^{5}
\end{aligned}
$$

where $a_{1}, a_{2}, a_{3}, a_{4}, a_{5}, b_{1}, b_{2}, b_{3}, b_{4}, c_{1}, c_{2}, c_{3}, \delta_{1}, \delta_{2}, e, h_{1}, h_{2}, h_{3}, h_{4}, h_{5}, h_{6}, k_{1}, k_{2}, k_{3}$ are functions on $P$ uniquely defined by the equivalence class of forms (49).

The system (50)-(51) provides all the local invariants for the equivalence class of forms (49) satisfying $F_{q q} \neq 0$. If one is given two triples of 1 -forms

$$
\begin{gathered}
\omega^{1}=\mathrm{d} z-F(x, y, p, q, z) \mathrm{d} x, \quad F_{q q} \neq 0 \\
\omega^{2}=\mathrm{d} y-p \mathrm{~d} x \\
\omega^{3}=\mathrm{d} p-q \mathrm{~d} x
\end{gathered}
$$

and

$$
\begin{gathered}
\bar{\omega}^{1}=\mathrm{d} \bar{z}-\bar{F}(\bar{x}, \bar{y}, \bar{p}, \bar{q}, \bar{z}) \mathrm{d} \bar{x}, \quad \bar{F}_{\bar{q} \bar{q}} \neq 0, \\
\bar{\omega}^{2}=\mathrm{d} \bar{y}-\bar{p} \mathrm{~d} \bar{x}
\end{gathered}
$$




$$
\bar{\omega}^{3}=\mathrm{d} \bar{p}-\bar{q} \mathrm{~d} \bar{x}
$$

on respective manifolds $J$ and $\bar{J}$ parametrized by $(x, y, p, q, z)$ and $(\bar{x}, \bar{y}, \bar{p}, \bar{q}, \bar{z})$, then there exists a local diffeomorphism

$$
\left(\begin{array}{l}
x \\
y \\
p \\
q \\
z
\end{array}\right) \stackrel{\phi}{\rightarrow}\left(\begin{array}{c}
\bar{x} \\
\bar{y} \\
\bar{p} \\
\bar{q} \\
\bar{z}
\end{array}\right)=\left(\begin{array}{l}
\bar{x}(x, y, p, q, z) \\
\bar{y}(x, y, p, q, z) \\
\bar{p}(x, y, p, q, z) \\
\bar{q}(x, y, p, q, z) \\
\bar{z}(x, y, p, q, z)
\end{array}\right)
$$

realizing

$$
\begin{aligned}
& \phi^{*}\left(\bar{\omega}^{1}\right)=\alpha \omega^{1}+\beta \omega^{2}+\gamma \omega^{3} \\
& \phi^{*}\left(\bar{\omega}^{2}\right)=\delta \omega^{1}+\epsilon \omega^{2}+\lambda \omega^{3} \\
& \phi^{*}\left(\bar{\omega}^{3}\right)=\kappa \omega^{1}+\mu \omega^{2}+\nu \omega^{3}
\end{aligned}
$$

iff there exists a diffeomorphism $\Phi: P \rightarrow \bar{P}$ between the associated 14-dimensional manifolds $P$ and $\bar{P}$ of Theorem 8 such that

$$
\Phi^{*}\left(\bar{\theta}^{i}\right)=\theta^{i}, \quad \Phi^{*}\left(\bar{\Omega}_{\mu}\right)=\Omega_{\mu}
$$

for all $i=1,2,3,4,5$ and $\mu=1,2,3, \ldots, 9$. This, in particular means that to realize the equivalence between the $\left(\omega^{i}\right) \mathrm{s}$ and $\left(\bar{\omega}^{i}\right) \mathrm{s}$, the diffeomorphism $\Phi$ must also satisfy

$$
\Phi^{*}\left(\bar{a}_{1}\right)=a_{1}, \quad \Phi^{*}\left(\bar{b}_{1}\right)=b_{1}, \quad \Phi^{*}\left(\bar{c}_{1}\right)=c_{1}, \quad \text { etc. }
$$

This gives severe algebraic (i.e. non-differential) constraints on $\Phi$ and, in generic cases, quickly leads to the answer if the two systems of forms $\left(\omega^{i}\right)$ and $\left(\bar{\omega}^{i}\right)$ are equivalent.

In view of the above we ask for those equivalence classes of forms (49) which correspond to systems (501)-(51) with all the scalar invariants $\left(a_{1}, a_{2}, a_{3}, a_{4}, a_{5}, b_{1}, b_{2}, b_{3}, b_{4}, c_{1}, c_{2}, c_{3}, \delta_{1}, \delta_{2}, e, h_{1}, h_{2}, h_{3}, h_{4}\right.$, $\left.h_{5}, h_{6}, k_{1}, k_{2}, k_{3}\right)$ being constants. It follows that it is possible if and only if all of them are identically equal to zero. In this well defined case the system (150)-(51) can be understood as a system consisting of right invariants forms $\left(\theta^{i}, \Omega_{\mu}\right)$ on a 14-dimensional Lie group. This group is simple and has indefinite Killing form, as can be seen from the structure constant coefficients defined by the system (50)-(51) with all the scalar invariants vanishing. This identifies this group as a noncompact real form $\tilde{G}_{2}$ of the exceptional group $G_{2}$.

It follows that there is only one equivalence class of forms (49) corresponding to the system (50)-(151) with all the scalar invariants vanishing. It can be defined by the function

$$
F=q^{2}
$$

associated with the Hilbert equation

$$
z^{\prime}=\left(y^{\prime \prime}\right)^{2}
$$

In case of general scalar invariants, the system (50)-(51) defines a curvature of a certain Cartan $\tilde{\mathbf{g}}_{2}$-valued connection which 'measures' how much the equivalence class of forms (49) is distorted from the flat Hilbert case corresponding to $F=q^{2}$. To define this connection we first observe that the system (150)-(51) guarantees that $P$ is foliated by 9-dimensional leaves. These are the integral manifolds of the distribution spanned by vector fields $Y_{\mu}, \mu=1,2, \ldots 9$ which, together with $X_{i}$, $i=1,2, \ldots 5$, form a frame $\left(X_{1}, X_{2}, X_{3}, X_{4}, X_{5}, Y_{1}, Y_{2}, Y_{3}, Y_{4}, Y_{5}, Y_{6}, Y_{7}, Y_{8}, Y_{9}\right)$ dual to the invariant 
coframe $\left(\theta^{1}, \theta^{2}, \theta^{3}, \theta^{4}, \theta^{5}, \Omega_{1}, \Omega_{2}, \Omega_{3}, \Omega_{4}, \Omega_{5}, \Omega_{6}, \Omega_{7}, \Omega_{8}, \Omega_{9}\right)$ on $P$. (The fact that this distribution is integrable, is a simple corollary, from equations (50), which show that the basis $\theta^{i}, i=1,2, . .5$, of its annihilator is a differential ideal). This proves that the manifold $P$ is fibered over a 5 -dimensional space of leaves of this distribution. This space may be identified with the $(x, y, p, q, z)$ space $J$ on which the original forms $\omega^{i}, i=1,2, \ldots 5$, defining the equivalence class (49) reside. Thus we have a fibration $P \rightarrow J$, which is actually a principal fibre bundle with the 9-dimensional parabolic subgroup $H$ of $\tilde{G}_{2}$ as its structure group. On this fibre bundle the following matrix of 1-forms:

$$
\omega_{\tilde{G}_{2}}=\left(\begin{array}{ccccccc}
-\Omega_{1}-\Omega_{4} & -\Omega_{8} & -\Omega_{9} & -\frac{1}{\sqrt{3}} \Omega_{7} & \frac{1}{3} \Omega_{5} & \frac{1}{3} \Omega_{6} & 0 \\
\theta^{1} & \Omega_{1} & \Omega_{2} & \frac{1}{\sqrt{3}} \theta^{4} & -\frac{1}{3} \theta^{3} & 0 & \frac{1}{3} \Omega_{6} \\
\theta^{2} & \Omega_{3} & \Omega_{4} & \frac{1}{\sqrt{3}} \theta^{5} & 0 & -\frac{1}{3} \theta^{3} & -\frac{1}{3} \Omega_{5} \\
\frac{2}{\sqrt{3}} \theta^{3} & \frac{2}{\sqrt{3}} \Omega_{5} & \frac{2}{\sqrt{3}} \Omega_{6} & 0 & \frac{1}{\sqrt{3}} \theta^{5} & -\frac{1}{\sqrt{3}} \theta^{4} & -\frac{1}{\sqrt{3}} \Omega_{7} \\
\theta^{4} & \Omega_{7} & 0 & \frac{2}{\sqrt{3}} \Omega_{6} & -\Omega_{4} & \Omega_{2} & \Omega_{9} \\
\theta^{5} & 0 & \Omega_{7} & -\frac{2}{\sqrt{3}} \Omega_{5} & \Omega_{3} & -\Omega_{1} & -\Omega_{8} \\
0 & \theta^{5} & -\theta^{4} & \frac{2}{\sqrt{3}} \theta^{3} & -\theta^{2} & \theta^{1} & \Omega_{1}+\Omega_{4}
\end{array}\right),
$$

becomes a Cartan connection with values in the Lie algebra of $\tilde{G}_{2}$. (The fact that $\omega_{\tilde{G}_{2}}$ is $\tilde{\mathbf{g}}_{2^{-}}$ valued can be checked e.g. by successive replacement of one of the 14 forms $\left(\theta^{1}, \theta^{2}, \theta^{3}, \theta^{4}, \theta^{5}, \overline{\Omega_{1}}\right.$, $\left.\Omega_{2}, \Omega_{3}, \Omega_{4}, \Omega_{5}, \Omega_{6}, \Omega_{7}, \Omega_{8}, \Omega_{9}\right)$ in $\omega_{\tilde{G}_{2}}$ by 1 with simultaneous replacement of all the others forms by 0 . The so obtained 14 matrices satisfy the commutation relations of $\underline{\tilde{g}_{2}}$.) The curvature of this connection

$$
\mathcal{R}=\mathrm{d} \omega_{\tilde{G}_{2}}+\omega_{\tilde{G}_{2}} \wedge \omega_{\tilde{G}_{2}},
$$

being horizontal, involves only $\theta^{i} \wedge \theta^{j}$ terms. This when compared with equations (51), enables the scalar invariants to be interpreted as the curvature coefficients of $\omega_{\tilde{G}_{2}}$.

Another interpretation of $\omega_{\tilde{G}_{2}}$ can be obtained by recalling that $\tilde{G}_{2}$ is naturally embedded in $\mathbf{S O}(4,3)$ as its subgroup stabilizing a generic 3 -form in $\mathbf{R}^{(4,3)}$. We have chosen a 7 -dimensional representation of the Lie algebra $\tilde{\mathbf{g}}_{2}$ in such a way that the connection $\omega_{\tilde{G}_{2}}$ can be interpreted as a reduction of a Cartan normal conformal connection associated with a certain $(3,2)$-signature conformal metric defined on $J$. In the following we describe this view point.

Given an equivalence class of forms (49) satisfying $F_{q q} \neq 0$ and using the forms $\left(\theta^{1}, \theta^{2}, \theta^{3}, \theta^{4}, \theta^{5}\right)$ associated with them via Theorem 8 we define a following bilinear form

$$
\tilde{g}=2 \theta^{1} \theta^{5}-2 \theta^{2} \theta^{4}+\frac{4}{3} \theta^{3} \theta^{3}
$$

on $P$. This form is clearly degenerate and has signature $(+,+,+,-,-, 0,0,0,0,0,0,0,0,0)$. Using the frame $\left(X_{1}, X_{2}, X_{3}, X_{4}, X_{5}, Y_{1}, Y_{2}, Y_{3}, Y_{4}, Y_{5}, Y_{6}, Y_{7}, Y_{8}, Y_{9}\right)$ on $P$ defined above, we see that the degenerate directions of $\tilde{g}$ are tangent to the vectors $Y_{\mu}$. Now, the system (150) guarantees that the form $\tilde{g}$ scales when Lie dragged along any of the directions $Y_{\mu}$. In other words we have

$$
\mathcal{L}_{Y_{\mu}} \tilde{g}=\lambda_{\mu} \tilde{g}
$$


with some functions $\lambda_{\mu}$. This, when compared with the fact that the distribution spanned by $Y_{\mu}$, $\mu=1,2, \ldots 9$, defines a foliation on $P$, means that the degenerate bilinear form $\tilde{g}$ projects from $P$ to $J$, the space of leaves of this foliation, defining there a conformal metric $\left[G_{(3,2)}\right]$ of signature $(+,+,+,-,-)$. It is this conformal structure that yields all the information about the local invariants of an equivalence class of forms (49). Calculating the Cartan normal conformal connection of this conformal structure, leads to the conclusion that it is reducible to the $\tilde{\mathbf{g}}_{2}$-valued Cartan connection $\omega_{\tilde{G}_{2}}$ on $P$.

Remarkably the conformal metric $\left[G_{(3,2)}\right]$ is defined on the same space $J$ on which the original forms $\omega^{i}, i=1,2, \ldots 5$, defining the equivalence class (49) were defined. Thus, it is possible to write down a local representative $G_{(3,2)}$ of $\left[G_{(3,2)}\right]$ in coordinates $(x, y, p, q, z)$ in which the forms $\omega^{i}$ read

$$
\begin{gathered}
\omega^{1}=\mathrm{d} z-F(x, y, p, q, z) \mathrm{d} x \\
\omega^{2}=\mathrm{d} y-p \mathrm{~d} x \\
\omega^{3}=\mathrm{d} p-q \mathrm{~d} x \\
\omega^{4}=\mathrm{d} q \\
\omega^{5}=\mathrm{d} x .
\end{gathered}
$$

Introducing the total differential operator $D$ on $J$ by

$$
D=\partial_{x}+p \partial_{y}+q \partial_{p}+F \partial_{z}
$$

we find that a representative of $\left[G_{(3,2)}\right]$ is given by

$$
\begin{aligned}
& G_{(3,2)}= \\
& {\left[D F_{q q}^{2} F_{q q}^{2}+6 D F_{q} D F_{q q q} F_{q q}^{2}-6 D F_{q q q} F_{p} F_{q q}^{2}-3 D D F_{q q} F_{q q}^{3}+9 D F_{q p} F_{q q}^{3}-9 F_{p p} F_{q q}^{3}+\right.} \\
& 9 D F_{q z} F_{q} F_{q q}^{3}-18 F_{p z} F_{q} F_{q q}^{3}+3 D F_{z} F_{q q}^{4}-6 D F_{q} F_{q q}^{2} F_{q q p}+6 F_{p} F_{q q}^{2} F_{q q p}- \\
& 8 D F_{q} D F_{q q} F_{q q} F_{q q q}+8 D F_{q q} F_{p} F_{q q} F_{q q q}+3 D D F_{q} F_{q q}^{2} F_{q q q}-3 D F_{p} F_{q q}^{2} F_{q q q}-3 D F_{z} F_{q} F_{q q}^{2} F_{q q q}+ \\
& 4\left(D F_{q}\right)^{2} F_{q q q}^{2}-8 D F_{q} F_{p} F_{q q q}^{2}-3\left(D F_{q}\right)^{2} F_{q q} F_{q q q q}+4 F_{p}^{2} F_{q q q}^{2}+6 D F_{q} F_{p} F_{q q} F_{q q q q}- \\
& 3 F_{p}^{2} F_{q q} F_{q q q q}-6 D F_{q} F_{q} F_{q q}^{2} F_{q q z}+6 F_{p} F_{q} F_{q q}^{2} F_{q q z}-3 D F_{q} F_{q q}^{3} F_{q z}+12 F_{p} F_{q q}^{3} F_{q z}+ \\
& 3 F_{q q}^{2} F_{q q q} F_{y}-6 D F_{q q q} F_{q} F_{q q}^{2} F_{z}+4 D F_{q q} F_{q q}^{3} F_{z}+6 F_{q} F_{q q}^{2} F_{q q p} F_{z}+8 D F_{q q} F_{q} F_{q q} F_{q q q} F_{z}- \\
& 4 D F_{q} F_{q q}^{2} F_{q q q} F_{z}-9 F_{q p} F_{q q}^{3} F_{z}+F_{p} F_{q q}^{2} F_{q q q} F_{z}-8 D F_{q} F_{q} F_{q q q}^{2} F_{z}+8 F_{p} F_{q} F_{q q q}^{2} F_{z}+ \\
& 6 D F_{q} F_{q} F_{q q} F_{q q q q} F_{z}-6 F_{p} F_{q} F_{q q} F_{q q q q} F_{z}+18 F_{q q}^{3} F_{q y}+6 F_{q}^{2} F_{q q}^{2} F_{q q z} F_{z}+3 F_{q} F_{q q}^{3} F_{q z} F_{z}- \\
& \left.2 F_{q q}^{4} F_{z}^{2}+F_{q} F_{q q}^{2} F_{q q q} F_{z}^{2}+4 F_{q}^{2} F_{q q q}^{2} F_{z}^{2}-3 F_{q}^{2} F_{q q} F_{q q q q} F_{z}^{2}-9 F_{q}^{2} F_{q q}^{3} F_{z z}\right]\left(\tilde{\omega}^{1}\right)^{2}+ \\
& {\left[6 D F_{q q q} F_{q q}^{2}-6 F_{q q}^{2} F_{q q p}-8 D F_{q q} F_{q q} F_{q q q}+8 D F_{q} F_{q q q}^{2}-8 F_{p} F_{q q q}^{2}-6 D F_{q} F_{q q} F_{q q q q}+\right.} \\
& \left.6 F_{p} F_{q q} F_{q q q q}-6 F_{q} F_{q q}^{2} F_{q q z}+6 F_{q q}^{3} F_{q z}+2 F_{q q}^{2} F_{q q q} F_{z}-8 F_{q} F_{q q q}^{2} F_{z}+6 F_{q} F_{q q} F_{q q q q} F_{z}\right] \tilde{\omega}^{1} \tilde{\omega}^{2}+ \\
& {\left[10 D F_{q q} F_{q q}^{3}-10 D F_{q} F_{q q}^{2} F_{q q q}+10 F_{p} F_{q q}^{2} F_{q q q}-10 F_{q q}^{4} F_{z}+10 F_{q} F_{q q}^{2} F_{q q q} F_{z}\right] \tilde{\omega}^{1} \tilde{\omega}^{3}+} \\
& 30 F_{q q}^{4} \tilde{\omega}^{1} \tilde{\omega}^{4}+\left[30 D F_{q} F_{q q}^{3}-30 F_{p} F_{q q}^{3}-30 F_{q} F_{q q}^{3} F_{z}\right] \tilde{\omega}^{1} \tilde{\omega}^{5}+ \\
& {\left[4 F_{q q q}^{2}-3 F_{q q} F_{q q q q}\right]\left(\tilde{\omega}^{2}\right)^{2}-10 F_{q q}^{2} F_{q q q} \tilde{\omega}^{2} \tilde{\omega}^{3}+30 F_{q q}^{3} \tilde{\omega}^{2} \tilde{\omega}^{5}-20 F_{q q}^{4}\left(\tilde{\omega}^{3}\right)^{2} \text {, }}
\end{aligned}
$$

where the tilded omegas are defined by

$$
\begin{gathered}
\tilde{\omega}^{1}=\mathrm{d} y-p \mathrm{~d} x \\
\tilde{\omega}^{2}=\mathrm{d} z-F \mathrm{~d} x-F_{q}(\mathrm{~d} p-q \mathrm{~d} x) \\
\tilde{\omega}^{3}=\mathrm{d} p-q \mathrm{~d} x \\
\tilde{\omega}^{4}=\mathrm{d} q \\
\tilde{\omega}^{5}=\mathrm{d} x .
\end{gathered}
$$


Despite of its ugliness this formula may be useful if one wants to write down the Cartan invariant forms $\left(\theta^{i}, \Omega_{\mu}\right)$ and the scalar invariants $a_{1}, a_{2}, \ldots$ directly in terms of the function $F=$ $F\left(x, y, y^{\prime}, y^{\prime \prime}, z\right)$ and its derivatives.

We can summarize the above considerations in the following theorem.

\section{Theorem 9}

The conformal class of $(3,2)$-signature metrics $G_{(3,2)}$ which are naturally defined on the $J$ space parametrized by $(x, y, p, q, z)$ encodes an invariant information about a class of forms

$$
\begin{gathered}
\omega^{1}=\mathrm{d} z-F(x, y, p, q, z) \mathrm{d} x \\
\omega^{2}=\mathrm{d} y-p \mathrm{~d} x \\
\omega^{3}=\mathrm{d} p-q \mathrm{~d} x \\
\omega^{4}=\mathrm{d} q \\
\omega^{5}=\mathrm{d} x .
\end{gathered}
$$

associated with a second order Monge equation

$$
z^{\prime}=F\left(x, y, y^{\prime}, y^{\prime \prime}, z\right)
$$

satisfying $F_{q q} \neq 0$.

Among all 5-dimensional $(3,2)$-signature metrics the metrics $G_{(3,2)}$ are distinguished by the requirement that their $\underline{\mathbf{s o}}(4,3)$-valued Cartan normal conformal connection is reducible to a $\underline{\mathbf{g}}_{2}$-valued Cartan connection $\omega_{\tilde{G}_{2}}$.

Interestingly the conformal metrics $G_{(3,2)}$ are very rarely conformal to Einstein metrics. Even weaker curvature conditions, which are necessary for a metric to be conformal to Einstein, such as e.g. conformal C-space conditions (see Ref. [1] for the definition), are not always satisfied by the metrics $G_{(2,3)}$. However there are examples of the second order Monge equations which correspond to the conformally Einstein metrics $G_{(3,2)}$. Below, we present one of such examples.

\section{Example 6}

Consider a second order Monge equation

$$
z^{\prime}=F\left(y^{\prime \prime}\right), \quad \text { with } \quad F_{y^{\prime \prime} y^{\prime \prime}} \neq=0 .
$$

Since $F$ depends on only one variable $q$ we will denote its derivatives by $F_{q}=F^{\prime}$, etc. Its corresponding forms on $J$ are $\omega^{1}=\mathrm{d} z-F(q) \mathrm{d} x, \omega^{2}=\mathrm{d} y-p \mathrm{~d} x, \omega^{3}=\mathrm{d} p-q \mathrm{~d} x, \omega^{4}=\mathrm{d} q, \omega^{5}=\mathrm{d} x$, with the tilded forms appearing in (54)given by

$$
\begin{gathered}
\tilde{\omega}^{1}=\mathrm{d} y-p \mathrm{~d} x \\
\tilde{\omega}^{2}=\mathrm{d} z-F \mathrm{~d} x-F^{\prime}(\mathrm{d} p-q \mathrm{~d} x) \\
\tilde{\omega}^{3}=\mathrm{d} p-q \mathrm{~d} x \\
\tilde{\omega}^{4}=\mathrm{d} q \\
\tilde{\omega}^{5}=\mathrm{d} x .
\end{gathered}
$$

The invariant forms $\left(\theta^{1}, \theta^{2}, \theta^{3}, \theta^{4}, \theta^{5}, \Omega_{1}, \Omega_{2}, \Omega_{3}, \Omega_{4}, \Omega_{5}, \Omega_{6}, \Omega_{7}, \Omega_{8}, \Omega_{9}\right)$ of theorem 8 are totally determined by forms $\left(\theta^{1}, \theta^{2}, \theta^{3}, \theta^{4}, \theta^{5}, \Omega_{1}, \Omega_{2}, \Omega_{3}, \Omega_{4}, \Omega_{5}, \Omega_{6}, \Omega_{7}, \Omega_{8}, \Omega_{9}\right)$ on $J$ which satisfy system (50)- 
(51). Staring with (55) we find that on $J$ these forms can be represented by

$$
\begin{gathered}
\theta^{1}=\tilde{\omega}^{1} \\
\theta^{2}=\tilde{\omega}^{2} \\
\theta^{3}=-\left(F^{\prime \prime}\right)^{\frac{1}{3}} \tilde{\omega}^{3} \\
\theta^{4}=\left(F^{\prime \prime}\right)^{-\frac{1}{3}}\left[\tilde{\omega}^{5}-\frac{1}{3} F^{(3)}\left(F^{\prime \prime}\right)^{-1} \tilde{\omega}^{3}+\frac{1}{30}\left(-3 F^{\prime \prime} F^{(4)}+4 F^{(3) 2}\right)\left(F^{\prime \prime}\right)^{-3} \tilde{\omega}^{2}\right] \\
\theta^{5}=-\left(F^{\prime \prime}\right)^{\frac{2}{3}} \tilde{\omega}^{4}, \\
\Omega_{1}=0 \\
\Omega_{2}=\frac{1}{90}\left[-45 F^{\prime \prime} F^{(3)} F^{(4)}+40 F^{(3) 3}+9\left(F^{\prime \prime}\right)^{2} F^{(5)}\right]\left(F^{\prime \prime}\right)^{-5} \theta^{2}+\frac{1}{30}\left[-3 F^{\prime \prime} F^{(4)}+4 F^{(3) 2}\right]\left(F^{\prime \prime}\right)^{-\frac{10}{3}} \theta^{3} \\
\Omega_{3}=0, \quad \Omega_{4}=0, \quad \Omega_{5}=0, \\
\Omega_{6}=-\frac{1}{30}\left[-3 F^{\prime \prime} F^{(4)}+4 F^{(3) 2}\right]\left(F^{\prime \prime}\right)^{-\frac{10}{3}} \theta^{5} \\
\Omega_{7}=0, \quad \Omega_{8}=0, \quad \Omega_{9}=0 .
\end{gathered}
$$

In this setting the only nonvanishing function among $\left(a_{1}, a_{2}, a_{3}, a_{4}, a_{5}, b_{1}, b_{2}, b_{3}, b_{4}, c_{1}, c_{2}, c_{3}, \delta_{1}, \delta_{2}, e\right.$, $\left.h_{1}, h_{2}, h_{3}, h_{4}, h_{5}, h_{6}, k_{1}, k_{2}, k_{3}\right)$ is

$a_{5}=\left\{-224 F^{(3) 4}+336 F^{\prime \prime} F^{(3) 2} F^{(4)}-80\left(F^{\prime \prime}\right)^{2} F^{(3)} F^{(5)}+\left(F^{\prime \prime}\right)^{2}\left[-51 F^{(4) 2}+10 F^{\prime \prime} F^{(6)}\right]\right\} /\left[100\left(F^{\prime \prime}\right)^{\frac{20}{3}}\right]$.

Now applying formula (53) to the forms (56), or using formula (54) for $F=F(q)$, we get the following representative for the metrics $\left[G_{(3,2)}\right]$ :

$$
\begin{gathered}
G_{(3,2)}=30\left(F^{\prime \prime}\right)^{4}[\mathrm{~d} q \mathrm{~d} y-p \mathrm{~d} q \mathrm{~d} x]+\left[4 F^{(3) 2}-3 F^{\prime \prime} F^{(4)}\right] \mathrm{d} z^{2}+ \\
2\left[-5\left(F^{\prime \prime}\right)^{2} F^{(3)}-4 F^{\prime} F^{(3) 2}+3 F^{\prime} F^{\prime \prime} F^{(4)}\right] \mathrm{d} p \mathrm{~d} z+ \\
2\left[15\left(F^{\prime \prime}\right)^{3}+5 q\left(F^{\prime \prime}\right)^{2} F^{(3)}-4 F F^{(3) 2}+4 q F^{\prime} F^{(3) 2}+3 F F^{\prime \prime} F^{(4)}-3 q F^{\prime} F^{\prime \prime} F^{(4)}\right] \mathrm{d} x \mathrm{~d} z+ \\
{\left[-20\left(F^{\prime \prime}\right)^{4}+10 F^{\prime}\left(F^{\prime \prime}\right)^{2} F^{(3)}+4\left(F^{\prime}\right)^{2} F^{(3) 2}-3\left(F^{\prime}\right)^{2} F^{\prime \prime} F^{(4)}\right] \mathrm{d} p^{2}+} \\
2\left[-15 F^{\prime}\left(F^{\prime \prime}\right)^{3}+20 q\left(F^{\prime \prime}\right)^{4}+5 F\left(F^{\prime \prime}\right)^{2} F^{(3)}-10 q F^{\prime}\left(F^{\prime \prime}\right)^{2} F^{(3)}+\right. \\
\left.4 F F^{\prime} F^{(3) 2}-4 q\left(F^{\prime}\right)^{2} F^{(3) 2}-3 F F^{\prime} F^{\prime \prime} F^{(4)}+3 q\left(F^{\prime}\right)^{2} F^{\prime \prime} F^{(4)}\right] \mathrm{d} p \mathrm{~d} x+ \\
{\left[-30 F\left(F^{\prime \prime}\right)^{3}+30 q F^{\prime}\left(F^{\prime \prime}\right)^{3}-20 q^{2}\left(F^{\prime \prime}\right)^{4}-10 q F\left(F^{\prime \prime}\right)^{2} F^{(3)}+10 q^{2} F^{\prime}\left(F^{\prime \prime}\right)^{2} F^{(3)}+4 F^{2} F^{(3) 2}-\right.} \\
\left.8 q F F^{\prime} F^{(3) 2}+4 q^{2}\left(F^{\prime}\right)^{2} F^{(3) 2}-3 F^{2} F^{\prime \prime} F^{(4)}+6 q F F^{\prime} F^{\prime \prime} F^{(4)}-3 q^{2}\left(F^{\prime}\right)^{2} F^{\prime \prime} F^{(4)}\right] \mathrm{d} x^{2} .
\end{gathered}
$$

It is a matter of checking that this metric is conformal to an Einstein metric $g=\mathrm{e}^{2 \Upsilon} G_{(3,2)}$ with the conformal factor $\Upsilon=\Upsilon(q)$ satisfying equation

$$
10\left(F^{\prime \prime}\right)^{2}\left[\Upsilon^{\prime \prime}-\left(\Upsilon^{\prime}\right)^{2}\right]-40 F^{\prime \prime} F^{(3)} \Upsilon^{\prime}+17 F^{\prime \prime} F^{(4)}-56 F^{(3) 2}=0 .
$$

Cartan 4] classified various types of nonequivalent forms (49) according to the roots of the polynomial

$$
\Psi(z)=a_{1} z^{4}+4 a_{2} z^{3}+6 a_{3} z^{2}+4 a_{4} z+a_{5},
$$

where $\left(a_{1}, a_{2}, a_{3}, a_{4}, a_{5}\right)$ are the scalar invariants given by (51). This polynomial encodes partial ${ }^{5}$ information of the Weyl tensor of the associated metrics $G_{(3,2)}$. In particular, its invariant $I_{\Psi}=$ $6 a_{3}^{2}-8 a_{2} a_{4}+2 a_{1} a_{5}$ is, modulo a numerical factor, proportional to the square of the Weyl tensor $C^{2}=C^{\mu \nu \rho \sigma} C_{\mu \nu \rho \sigma}$ of the metric $G_{(3,2)}$. Vanishing of $I_{\Psi}$ means that $\Psi=\Psi(z)$ has a root with multiplicity no smaller than 3 . Our example above corresponds to the situation when this multiplicity

\footnotetext{
${ }^{5}$ For completeness we give the exact formula for The Weyl tensor of metrics $G(3,2)$ in the Appendix
} 
is equal to 4. According to Cartan 4 all nonequivalent forms for which $\Psi$ has quartic root are covered by this example. The nonequivalent classes are distinguished by the only nonvanishing scalar invariant $a_{5}$ of (57), to which the Weyl tensor of metric (58) is proportional.

We were unable to construct an example of forms (49) for which $\Psi$ has precisely triple root. For this it is enough to assume that among the scalar invariants $\left(a_{1}, a_{2}, a_{3}, a_{4}, a_{5}\right)$ only $a_{4}$ is nonvanishing. In such situation Cartan shows that the system (50)-(51) reduces to an invariant coframe on $J$. Despite the fact that in this case the system is reducible to 5 -dimensions it is difficult, to find nonhomogeneous examples of forms which satisfy it.

\section{Acknowledgements}

This work was inspired by discussions on the differential equations' aspect of the Null Surface Formulation of GR which I had with Ezra Ted Newman. It started during my stay at King's College London and was completed at the Institute for Mathematics of Humboldt University in Berlin, where I was a member of the VW Junior Research Group "Special Geometries in Mathematical Physics". I am very grateful to David C Robinson, Ilka Agricola and Thomas Friedrich for making the visits to these institutions possible and fruitful.

I acknowledge support from EPSRC grant GR/S34304/01 while at King's College London and the KBN grant 2 P03B 12724 while at Warsaw University.

\section{$7 \quad$ Appendix}

In the null coframe $\left(\alpha^{1}, \alpha^{2}, \alpha^{3}, \alpha^{4} \alpha^{5}\right)=\left(\theta^{1}, \theta^{2}, \frac{2 \sqrt{3}}{3} \theta^{3}, \theta^{4}, \theta^{5}\right)$ in which the metric (54) is

$$
G_{(3,2)}=2 \alpha^{1} \alpha^{5}-2 \alpha^{2} \alpha^{4}+\left(\alpha^{3}\right)^{2}
$$

the Weyl tensor 2-forms are:

$$
C_{\mu \nu}=\frac{1}{2} C_{\mu \nu \rho \sigma} \alpha^{\rho} \wedge \alpha^{\sigma}=\left(\begin{array}{ccccc}
0 & 0 & 0 & -w_{14} & w_{15} \\
0 & 0 & 0 & w_{15} & -w_{25} \\
0 & 0 & 0 & -w_{34} & w_{35} \\
w_{14} & -w_{15} & w_{34} & 0 & -w_{45} \\
-w_{15} & w_{25} & -w_{35} & w_{45} & 0
\end{array}\right),
$$

where

$$
\begin{aligned}
& w_{14}=\frac{3}{8} c_{3} \alpha^{1} \wedge \alpha^{2}+\frac{\sqrt{3}}{2} b_{3} \alpha^{1} \wedge \alpha^{3}+a_{3} \alpha^{1} \wedge \alpha^{4}+a_{4} \alpha^{1} \wedge \alpha^{5}+\frac{\sqrt{3}}{2} b_{4} \alpha^{2} \wedge \alpha^{3}+a_{4} \alpha^{2} \wedge \alpha^{4}+a_{5} \alpha^{2} \wedge \alpha^{5}, \\
& w_{15}=\frac{3}{8} c_{2} \alpha^{1} \wedge \alpha^{2}+\frac{\sqrt{3}}{2} b_{2} \alpha^{1} \wedge \alpha^{3}+a_{2} \alpha^{1} \wedge \alpha^{4}+a_{3} \alpha^{1} \wedge \alpha^{5}+\frac{\sqrt{3}}{2} b_{3} \alpha^{2} \wedge \alpha^{3}+a_{3} \alpha^{2} \wedge \alpha^{4}+a_{4} \alpha^{2} \wedge \alpha^{5}, \\
& w_{25}=\frac{3}{8} c_{1} \alpha^{1} \wedge \alpha^{2}+\frac{\sqrt{3}}{2} b_{1} \alpha^{1} \wedge \alpha^{3}+a_{1} \alpha^{1} \wedge \alpha^{4}+a_{2} \alpha^{1} \wedge \alpha^{5}+\frac{\sqrt{3}}{2} b_{2} \alpha^{2} \wedge \alpha^{3}+a_{2} \alpha^{2} \wedge \alpha^{4}+a_{3} \alpha^{2} \wedge \alpha^{5}, \\
& w_{34}=\frac{3 \sqrt{3}}{16} \delta_{2} \alpha^{1} \wedge \alpha^{2}+\frac{3}{4} c_{2} \alpha^{1} \wedge \alpha^{3}+\frac{\sqrt{3}}{2} b_{2} \alpha^{1} \wedge \alpha^{4}+\frac{\sqrt{3}}{2} b_{3} \alpha^{1} \wedge \alpha^{5}+\frac{3}{4} c_{3} \alpha^{2} \wedge \alpha^{3}+\frac{\sqrt{3}}{2} b_{3} \alpha^{2} \wedge \alpha^{4}+\frac{\sqrt{3}}{2} b_{4} \alpha^{2} \wedge \alpha^{5}, \\
& w_{35}=\frac{3 \sqrt{3}}{16} \delta_{1} \alpha^{1} \wedge \alpha^{2}+\frac{3}{4} c_{1} \alpha^{1} \wedge \alpha^{3}+\frac{\sqrt{3}}{2} b_{1} \alpha^{1} \wedge \alpha^{4}+\frac{\sqrt{3}}{2} b_{2} \alpha^{1} \wedge \alpha^{5}+\frac{3}{4} c_{2} \alpha^{2} \wedge \alpha^{3}+\frac{\sqrt{3}}{2} b_{2} \alpha^{2} \wedge \alpha^{4}+\frac{\sqrt{3}}{2} b_{3} \alpha^{2} \wedge \alpha^{5}, \\
& w_{45}=-\frac{9}{64} e \alpha^{1} \wedge \alpha^{2}+\frac{3 \sqrt{3}}{16} \delta_{1} \alpha^{1} \wedge \alpha^{3}+\frac{3}{8} c_{1} \alpha^{1} \wedge \alpha^{4}+\frac{3}{8} c_{2} \alpha^{1} \wedge \alpha^{5}+\frac{3 \sqrt{3}}{16} \delta_{2} \alpha^{2} \wedge \alpha^{3}+\frac{3}{8} c_{2} \alpha^{2} \wedge \alpha^{4}+\frac{3}{8} c_{3} \alpha^{2} \wedge \alpha^{5} .
\end{aligned}
$$




\section{References}

[1] Cartan E (1921) "Sur les espaces conformes generalises et l'universe optique" Comptes Rendus Acad. Sci. Paris 174, 857-859

[2] Cartan E (1924) "Varietes a connexion projective" Bull. Soc. Math. LII 205-241

[3] Cartan E (1941) "La geometria de las ecuaciones diferenciales de tercer orden" Rev. Mat. Hispano-Aamer. 4 1-31

[4] Cartan E (1910) "Les systemes de Pfaff a cinq variables et les equations aux derivees partielles du second ordre" Ann. Sc. Norm. Sup. 27 109-192

[5] Chern S S (1940) "The geometry of the differential equations $y^{\prime \prime \prime}=F\left(x, y, y^{\prime}, y^{\prime \prime}\right)$ " Sci. Rep. Nat. Tsing Hua Univ. 4 97-111

[6] Dunajski M, Mason L J, Tod K P, "Einstein-Weyl geometry, the dKP equation and twistor theory" J. Geom. Phys. 37 (2001), 63-93

[7] Fefferman C L (1976) "Monge-Ampere equations, the Bergman kernel, and geometry of pseudoconvex domains" Ann. of Math 103, 395-416, and correction ibid. 104, 393-394

[8] Fritelli S, Kozameh C N, Newman E T (1995) "GR via characteristic surfaces" J. Math. Phys. 36 4984-

[9] Fritelli S, Kozameh C, Newman E T (2001) "Differential geometry from differential equations" Comm. Math. Phys. 223 383-

[10] Fritelli S, Newman E T, Nurowski P (2003) "Conformal Lorentzian metrics on the spaces of curves and 2-surfaces" Class. Q. Grav. 20 3649-3659

[11] A R Gover, P Nurowski (2004) "Obstructions to conformally Einstein metrics" avaliable as math.DG/0405304 at http://xxx.lanl.gov

[12] Hilbert D (1912) "Ueber den Begriff der Klasse von Differentialgleichungen" Mathem. Annalen Bd. 73, 95-108

[13] Godlinski M, Nurowski P (in preparation) "Differential geometry of the third order ODEs"

[14] Kobayashi S (1972) Transformation Groups in Differential Geometry Springer, Berlin

[15] Newman E T, Nurowski P (2003) "Projective connections associated with second order ODEs" Class. Q. Grav. 20, 2325-2335

[16] Newman E T, Penrose R (1962) "An approach to gravitational radiation by a method of spin coefficients" J. Math. Phys. 3 566-

[17] Nurowski P, Sparling G A J (2003) "Three dimensional Cauchy-Riemann structures and second order ordinary differential equations" Class. Q. Grav. 20, 4995-5016

[18] Olver P J, (1996) Equivalence Invariants and Symetry (Cambridge University Press, Cambridge)

[19] Penrose R (1960) "A spinor approach to General Relativity" Ann. Phys. (NY) 10 171-201

[20] Penrose R (1967) "Twistor algebra” J. Math. Phys. 8 345-366 
[21] Petrov A Z (1954) "Classification of spaces defining gravitational fields" Sci. Not. Kazan State Univ. 114 55-69

[22] Sparling G A J (2003) private communications

[23] Tod K P (2000) "Einstein-Weyl spaces and third order differential equations" J. Math. Phys. $415572-$

[24] Wuenschmann K, (1905) "Ueber Beruhrungsbedingungen bei Differentialgleichungen", Dissertation, Greifswald. 